\title{
Merger of black hole and neutron star in general relativity: Tidal disruption, torus mass, and gravitational waves
}

\author{
Masaru Shibata \\ Graduate School of Arts and Sciences, University of Tokyo, Komaba, Meguro, Tokyo 153-8902, Japan \\ Keisuke Taniguchi \\ Department of Physics, University of Illinois at Urbana-Champaign, Illinois 61801, USA
}

\begin{abstract}
We systematically perform the merger simulation of black hole-neutron star (BH-NS) binaries in full general relativity, focusing on the case that the NS is tidally disrupted. We prepare BH-NS binaries in a quasicircular orbit as the initial condition in which the $\mathrm{BH}$ is modeled by a nonspinning moving puncture. For modeling the NS, we adopt the $\Gamma$-law equation of state with $\Gamma=2$ and the irrotational velocity field. We change the $\mathrm{BH}$ mass in the range $M_{\mathrm{BH}} \approx 3.3-4.6 M_{\odot}$, while the rest mass of the NS is fixed to be $M_{*}=1.4 M_{\odot}$ (i.e., the NS mass $\left.M_{\mathrm{NS}} \approx 1.3 M_{\odot}\right)$. The radius of the corresponding spherical NS is set in the range $R_{\mathrm{NS}} \approx 12-15 \mathrm{~km}$ (i.e., the compactness $\left.G M_{\mathrm{NS}} / R_{\mathrm{NS}} c^{2} \approx 0.13-0.16\right)$. We find for all the chosen initial conditions that the NS is tidally disrupted near the innermost stable circular orbit. For the model of $R_{\mathrm{NS}}=12 \mathrm{~km}$, more than $97 \%$ of the rest mass is quickly swallowed into the $\mathrm{BH}$ and the resultant torus mass surrounding the $\mathrm{BH}$ is less than $0.04 M_{\odot}$. For the model of $R_{\mathrm{NS}} \approx 14.7 \mathrm{~km}$, by contrast, the torus mass is about $0.16 M_{\odot}$ for the $\mathrm{BH}$ mass $\approx 4 M_{\odot}$. The thermal energy of the material in the torus increases by the shock heating occurred in the collision between the spiral arms, resulting in the temperature $10^{10}-10^{11}$ $\mathrm{K}$. Our results indicate that the merger between a low-mass BH and its companion NS may form a central engine of short gamma-ray bursts (SGRBs) of the total energy of order $10^{49}$ ergs if the compactness of the NS is fairly small $\lesssim 0.145$. However, for the canonical values $M_{\mathrm{NS}}=1.35 M_{\odot}$ and $R_{\mathrm{NS}}=12 \mathrm{~km}$, the merger results in small torus mass, and hence, it can be a candidate only for the low-energy SGRBs of total energy of order $10^{48} \mathrm{ergs}$. We also present gravitational waveforms during the inspiral, tidal disruption of the NS, and subsequent evolution of the disrupted material. We find that the amplitude of gravitational waves quickly decreases after the onset of tidal disruption. Although the quasinormal mode is excited, its gravitational wave amplitude is much smaller than that of the late inspiral phase. This reflects in the fact that the spectrum amplitude sharply falls above a cut-off frequency which is determined by the tidal disruption process. We also find that the cut-off frequency is 1.25-1.4 times larger than the frequency predicted by the study for the sequence of the quasicircular orbits and this factor of the deviation depends on the compactness of the NS.
\end{abstract}

PACS numbers: 04.25.Dm, 04.30.-w, 04.40.Dg

\section{INTRODUCTION}

The merger of black hole (BH)-neutron star (NS) binaries is one of the most promising sources of kilo-meter size laserinterferometric gravitational wave detectors such as LIGO and VIRGO. Although such system has not been observed yet in our Galaxy in contrast to NS-NS binaries [1], statistical studies based on the stellar evolution synthesis suggest that the merger will happen $1-10 \%$ as frequently as the merger of the NS-NS binaries in the universe 22]. Because the $\mathrm{BH}$ mass should be more than twice as large as the canonical NS mass $\sim 1.35 M_{\odot}$, the typical amplitude of gravitational waves from the BH-NS binaries will be larger than that from the NS-NS binaries even if the distance is larger. This indicates that the detection rate of the BH-NS binaries by the gravitational wave detectors may be more than $10 \%$ as high as that for the NS-NS binaries, suggesting that the detection of such system will be achieved in the near future.

The final fate of the BH-NS binaries is divided into two cases depending primarily on the $\mathrm{BH}$ mass: (1) when the BH mass is small enough, the NS will be tidally disrupted before it is swallowed by the $\mathrm{BH}$; (2) when the
BH mass is large enough, the NS will be swallowed into the $\mathrm{BH}$ without tidal disruption. The tidal disruption occurs when the tidal force of the BH is stronger than the self-gravity of the NS. Such condition is approximately written as

$$
\frac{M_{\mathrm{BH}} R_{\mathrm{NS}}}{r^{3}} \geq C^{2} \frac{M_{\mathrm{NS}}}{R_{\mathrm{NS}}^{2}},
$$

where $M_{\mathrm{NS}}$ is the NS mass, $R_{\mathrm{NS}}$ is the circumferential radius of the NS, $M_{\mathrm{BH}}$ is the $\mathrm{BH}$ mass, $r$ is the orbital separation, and $C$ is a nondimensional coefficient. Using the orbital angular velocity $\Omega$, Eq. (11) may be written as

$$
\Omega^{2} \frac{M_{\mathrm{BH}}}{M_{0}} \geq C^{2} \frac{G M_{\mathrm{NS}}}{R_{\mathrm{NS}}^{3}},
$$

where $G$ is the gravitational constant and $M_{0}=M_{\mathrm{BH}}+$ $M_{\mathrm{NS}}$. Actually, the latest high-precision numerical study for the quasicircular states of the BH-NS binaries shows that the tidal disruption occurs if the following condition is satisfied [3, 4]:

$$
\Omega \geq C\left(\frac{G M_{\mathrm{NS}}}{R_{\mathrm{NS}}^{3}}\right)^{1 / 2}\left(1+\frac{M_{\mathrm{NS}}}{M_{\mathrm{BH}}}\right)^{1 / 2}
$$


where the value of $C$ is $\approx 0.270$ for $\Gamma=2$ polytropic equation of state (EOS). We note that in $[3,4]$ the NS is assumed to be irrotational and the $\mathrm{BH}$ spin is set to be zero. According to an approximate general relativistic study [5], the value of $C$ depends weakly on the stiffness of the EOSs for $2 \leq \Gamma \leq 3$, and hence, it would be close to 0.27 for the NS which likely has such stiff EOS.

Substituting the typical values considered in this paper, the tidal disruption is expected to set in at

$$
\begin{aligned}
\frac{G M_{0} \Omega}{c^{3}}= & 0.0708\left(\frac{C}{0.270}\right)\left(\frac{M_{\mathrm{NS}}}{1.30 M_{\odot}}\right)^{3 / 2} \\
& \times\left(\frac{R_{\mathrm{NS}}}{13.0 \mathrm{~km}}\right)^{-3 / 2}\left(\frac{q}{1 / 3}\right)^{-1}\left(\frac{1+q}{4 / 3}\right)^{3 / 2}
\end{aligned}
$$

where $c$ is the speed of the light and $q$ denotes the mass ratio $q \equiv M_{\mathrm{NS}} / M_{\mathrm{BH}}$. The corresponding frequency of gravitational waves is calculated from $f \equiv \Omega / \pi$ as

$$
\begin{aligned}
f_{\text {tidal }}=8.79 & \times 10^{2} \mathrm{~Hz}\left(\frac{C}{0.270}\right)\left(\frac{M_{\mathrm{NS}}}{1.30 M_{\odot}}\right)^{1 / 2} \\
& \times\left(\frac{R_{\mathrm{NS}}}{13.0 \mathrm{~km}}\right)^{-3 / 2}\left(\frac{1+q}{4 / 3}\right)^{1 / 2} .
\end{aligned}
$$

According to the third post-Newtonian (PN) study, the innermost stable circular orbit (ISCO) for $q \approx 1 / 3$ is located at $G M_{0} \Omega / c^{3} \approx 0.11[6]$. The tidal effect reduces this value slightly to be $G M_{0} \Omega / c^{3} \sim 0.08-0.09[3$, 4] . Adopting this value, we expect that the tidal disruption of the NSs of mass $1.3 M_{\odot}$ occurs for $q \gtrsim 1 / 4$ if $R_{\mathrm{NS}}=13$ $\mathrm{km}$, and for $q \gtrsim 1 / 3$ if $R_{\mathrm{NS}}=11 \mathrm{~km}$. This indicates that the tidal disruption occurs only for the binaries of low-mass BH and NS at an orbit very close to the ISCO.

The tidal disruption of NSs by a $\mathrm{BH}$ has been studied with great interest because of the following reasons: (i) Gravitational waves during the tidal disruption may bring the information about the NS radius because the orbital frequency at the onset of tidal disruption depends strongly on the compactness of the NS $\left(G M_{\mathrm{NS}} / R_{\mathrm{NS}} c^{2}\right)$ $[3,4,45]$. The NS mass will be determined by the data analysis for observed gravitational waves in the inspiral phase [7]. If the NS radius could be determined from observed gravitational waves emitted during the tidal disruption, the resultant relation between the mass and the radius of the NSs may constrain the EOSs of the high density nuclear matter [8, 9]. (ii) The tidally disrupted NSs may form a disk or torus of mass larger than $0.01 M_{\odot}$ around the $\mathrm{BH}$ if the tidal disruption occurs outside the ISCO. Systems consisting of a rotating BH surrounded by a massive, hot torus have been proposed as one of the likely sources for the central engine of gamma-ray bursts with a short duration [10] (hereafter SGRBs). Hence, the merger of a low-mass $\mathrm{BH}$ and its companion NS can be a candidate central engine. According to the observational results by the Swift and HETE-2 satellites [11], the total energy of the SGRBs is larger than $\sim 10^{48} \mathrm{ergs}$, and typically $10^{49}-10^{50}$ ergs. The question is whether or not the mass and thermal energy of the torus are large enough for driving the SGRBs of such huge total energy.

The tidal disruption of NSs by a BH has been investigated in the Newtonian [12] and approximately general relativistic [13, 14] simulations. However, the criterion of the tidal disruption and the evolution of the tidally disrupted NS material would depend strongly on general relativistic effects around the $\mathrm{BH}$, and hence, a simulation in full general relativity is obviously required. In the previous paper [15], we presented our first numerical results for fully general relativistic simulation performed by our new code which had been improved from the code used for the NS-NS merger [16, 17]: We handle an orbiting $\mathrm{BH}$ employing the moving puncture method, which has been recently developed (e.g., 18, 19]). We prepared a quasicircular state composed of a nonspinning moving puncture $\mathrm{BH}$ and a corotating NS as the initial condition. We computed the BH-NS quasicircular state by employing our new formalism based on the moving puncture framework. From the merger simulation, we found that a torus with mass $\sim 0.1 M_{\odot}$ is an outcome for $M_{\mathrm{NS}}=1.3 M_{\odot}$ and $R_{\mathrm{NS}}=13-14 \mathrm{~km}$, and for $M_{\mathrm{BH}}=3-4 M_{\odot}$.

In the previous work, we assumed that the NSs are corotating around the center of mass of the system for simplicity. However, this is not very realistic velocity field for the NS in close compact binaries [20]. Recently, we have developed a new code for computing quasicircular states of a BH and an irrotational NS. For obtaining the irrotational velocity field of the NS, one has to solve the equation for the velocity potential [21]. We employ the same method as that for computing the NSNS binaries (e.g., 22, 23]), which has been used in our project for the NS-NS merger [16, 17]. (For solving the basic equations for the initial data, we use the spectral method library LORENE developed by the Meudon relativity group [24].) In this paper, we perform the simulations employing these new quasicircular states as the initial conditions. In addition, we modify our simulation code for the Einstein evolution equation to the fourthorder scheme for more accurate computation. Changing the $\mathrm{BH}$ mass and NS radius, we systematically investigate the dependence of the torus mass formed after the merger on $M_{\mathrm{BH}}$ and $R_{\mathrm{NS}}$. We also compute gravitational waveforms during the tidal disruption and their spectrum.

The paper is organized as follows. Section II summarizes the initial conditions chosen in this paper. Section III briefly describes the formulation and numerical methods for the simulation. Section IV presents the numerical results of the simulation for the merger of BH-NS binaries. Section V is devoted to a summary. In the following, the geometrical units of $c=G=1$ are used.

\section{INITIAL CONDITION}

We compute the initial condition for the numerical simulation by employing a formulation in the moving punc- 
TABLE I: Parameters for the quasicircular states of BH-NS binaries. The mass parameter of the puncture, the BH mass, the rest mass of the NS, the mass, radius, and normalized mass of the NS in isolation, the ADM mass of the system, the total angular momentum in units of $M^{2}$, the orbital period in units of $M_{0}=M_{\mathrm{BH}}+M_{\mathrm{NS}}$, and the compactness of the system defined by $\left(M_{0} \Omega\right)^{2 / 3}$. The BH mass is computed from the area of the apparent horizon $A$ as $(A / 16 \pi)^{1 / 2} . M_{0} \Omega$ is $\approx 0.040-0.041$ for all the models.

\begin{tabular}{cccccccccccc}
\hline Model & $M_{\mathrm{p}}\left(M_{\odot}\right)$ & $M_{\mathrm{BH}}\left(M_{\odot}\right)$ & $M_{*}\left(M_{\odot}\right)$ & $M_{\mathrm{NS}}\left(M_{\odot}\right)$ & $q$ & $R_{\mathrm{NS}}(\mathrm{km})$ & $M_{*} / \kappa^{1 / 2}$ & $M\left(M_{\odot}\right)$ & $J / M^{2}$ & $P_{0} / M_{0}$ & $\left(M_{0} \Omega\right)^{2 / 3}$ \\
\hline $\mathrm{A}$ & 3.906 & 3.975 & 1.400 & 1.302 & 0.327 & 13.2 & 0.150 & 5.227 & 0.662 & 154 & 0.118 \\
\hline $\mathrm{B}$ & 3.881 & 3.950 & 1.400 & 1.294 & 0.327 & 12.0 & 0.160 & 5.193 & 0.660 & 154 & 0.119 \\
\hline $\mathrm{C}$ & 3.930 & 4.001 & 1.400 & 1.310 & 0.328 & 14.7 & 0.140 & 5.260 & 0.663 & 152 & 0.119 \\
\hline $\mathrm{D}$ & 3.255 & 3.321 & 1.400 & 1.302 & 0.392 & 13.2 & 0.150 & 4.576 & 0.725 & 157 & 0.117 \\
\hline $\mathrm{E}$ & 3.234 & 3.300 & 1.400 & 1.294 & 0.392 & 12.0 & 0.160 & 4.546 & 0.721 & 154 & 0.118 \\
\hline $\mathrm{F}$ & 4.557 & 4.627 & 1.400 & 1.302 & 0.281 & 13.2 & 0.150 & 5.878 & 0.612 & 158 & 0.117 \\
\hline
\end{tabular}

ture framework which is described in our previous papers 15]. This formulation is slightly different from that in 3. 4, 25, 26], although both formulations are based on the confmormal flatness formulation for the three-metric, and solve the equations of the maximal slicing condition, and Hamiltonian and momentum constraints. (See 27] for the simulations preformed employing the initial data presented in [3]). Although the basic equations adopted in this paper are the same as those described in 15], we slightly change the method for defining the quasicircular state as follows: In the previous paper, we determined the center of mass of the system imposing that the dipole moment of the conformal factor at spatial infinity is zero. In this paper, the center of mass is determined from the condition that the azimuthal component of the shift vector $\beta^{\varphi}$ at the location of the puncture is equal to $-\Omega$ where $\Omega$ is the orbital angular velocity. Namely, we impose the corotating gauge condition at the location of the puncture. The reason for this change is that with this new method, the curve of the angular momentum $J$ as a function of the orbital angular velocity $\Omega$ along quasicircular sequences is closer to the curve derived from the third PN equation of motion [6]. Furthermore, the relation among the orbital angular velocity, ADM mass $M$, and angular momentum agrees with the results obtained by a different method [3, 4] fairly well; for the mass ratio and NS radii adopted in this paper, the disagreement of the angular momentum for a given angular velocity is $\sim 1 \%$.

As the velocity field of the NS, we assume the irrotational one because it is believed to be the realistic velocity field [20]. The density profile and velocity field are determined by solving the hydrostatic equation (the first integral of the Euler equation) and an elliptic-type equation for the velocity potential [21].

The NSs are modeled by a polytropic EOS

$$
P=\kappa \rho^{\Gamma},
$$

where $P$ is the pressure, $\rho$ the rest-mass density, $\kappa$ the polytropic constant, and $\Gamma$ the adiabatic index for which we set $\Gamma=2 . \kappa$ is a free parameter and in the following we choose it so as to fix the rest mass of the NSs to be $M_{*}=1.4 M_{\odot}$ because this value is close to the canonical value [1]. We note that the mass, radius, and time can be rescaled arbitrarily by changing $\kappa$ : i.e., if we change the value of $\kappa$ from $\kappa_{1}$ to $\kappa_{2}$, these quantities are rescaled systematically by a factor of $\left(\kappa_{2} / \kappa_{1}\right)^{1 / 2}$ for $\Gamma=2$. By contrast, the nondimensional parameters such as $M_{\mathrm{NS}} / R_{\mathrm{NS}}, M \Omega, M \kappa^{-1 / 2}$, and $R_{\mathrm{NS}} \kappa^{-1 / 2}$ are invariant. In this paper, We present the results for the specific values of $\kappa$ for the help to the general readers who are not familiar with the nondimensional units.

Because we choose $M_{*}=1.4 M_{\odot}$, the NS mass is in the narrow range $M_{\mathrm{NS}} \approx 1.29-1.31 M_{\odot}$ irrespective of the NS radius $R_{\mathrm{NS}}$. The NS radius is chosen to be 12.0, 13.2, and $14.7 \mathrm{~km}$. These values agree approximately with those predicted by the nuclear EOSs in which $R_{\mathrm{NS}} \approx 10-15 \mathrm{~km}$ for $M_{\mathrm{NS}}=1.3 M_{\odot}\left[28\right.$. The value $R_{\mathrm{NS}}=12 \mathrm{~km}$ agrees approximately with that predicted by the stiff nuclear EOSs such as the Akmal-Pandharipande-Ravenhall EOS [29].

The $\mathrm{BH}$ mass is chosen in the range $M_{\mathrm{BH}} \approx 3.3^{-}$ $4.6 M_{\odot}$. As we described in Sec. I, the tidal disruption of the NS occurs for such low-mass BH.

In the present work, we prepare the quasicircular states with $M_{0} \Omega \approx 0.04$. With such initial condition, the BHNS binary experiences about one and half orbits before the onset of merger. In order to reduce the spurious ellipticity and to take into account the nonzero radial velocity associated with the gravitational radiation reaction, we may need to perform a simulation for several orbits before the onset of merger, starting from a more distant orbit [30, 31, 32]. In the present paper, however, we focus primarily on the qualitative study of the tidal disruption events. For such purpose, the adopted initial conditions are acceptable. The simulation of the longterm evolution for the inspiral phase is left for the future.

In Table I, the parameters of the quasicircular states adopted in this paper are listed. Specifically, we prepare six models for clarifying the dependence of the tidal disruption events on the NS radius and mass ratio. The NSs for models A, B, and C and for models D and E, respectively, have approximately the same mass ratio $q=M_{\mathrm{NS}} / M_{\mathrm{BH}}$, although the NS radii are different each other. Comparing the numerical results among these models, the dependence of the properties of the tidal dis- 
ruption event on the NS radius is clarified. The NS radius for models A, D, and F and for models B and $\mathrm{E}$ are the same, although the mass ratios are different each other. Thus, comparison of the numerical results for these models clarifies the dependence of the properties of the tidal disruption event on the mass ratio.

\section{NUMERICAL METHODS}

The numerical code for the hydrodynamics is the same as that in [15] where we use a high-resolution central scheme with the third-order piece-wise parabolic interpolation and with a steep min-mod limiter in which the limiter parameter $b$ is set to be 2.5 (see appendix A of [34]). We have already used this numerical code for the simulation of the NS-NS binary merger [17, 33]. We adopt the $\Gamma$-law EOS in the simulation as

$$
P=(\Gamma-1) \rho \varepsilon,
$$

where $\varepsilon$ is the specific internal energy and $\Gamma=2$.

For solving the Einstein evolution equation, we use the original version of the BSSN formalism [35]. Namely, we evolve the conformal part of the three-metric $\phi \equiv$ $(\ln \gamma) / 12$, the trace part of the extrinsic curvature $K$, the conformal three-metric $\tilde{\gamma}_{i j} \equiv \gamma^{-1 / 3} \gamma_{i j}$, the tracefree extrinsic curvature $\tilde{A}_{i j} \equiv \gamma^{-1 / 3}\left(K_{i j}-K \gamma_{i j} / 3\right)$, and a three auxiliary variable $F_{i} \equiv \delta^{j k} \partial_{j} \tilde{\gamma}_{i k}$. Here, $\gamma_{i j}$ is the three-metric, $K_{i j}$ the extrinsic curvature, $\gamma \equiv \operatorname{det}\left(\gamma_{i j}\right)$, and $K \equiv K_{i j} \gamma^{i j}$. In the previous paper [15], we solved the equation for $\gamma^{-1 / 2}$. Since then, we have learned that we do not have to evolve the inverse of the conformal factor even in the moving puncture framework because we use the cell-centered grid in which the puncture is never located on the grid points.

For the condition of the lapse function $\alpha$ and the shift vector $\beta^{i}$, we adopt a dynamical gauge condition in the following form:

$$
\begin{aligned}
& \left(\partial_{t}-\beta^{i} \partial_{i}\right) \ln \alpha=-2 K, \\
& \partial_{t} \beta^{i}=0.75 \tilde{\gamma}^{i j}\left(F_{j}+\Delta t \partial_{t} F_{j}\right) .
\end{aligned}
$$

Here, $\Delta t$ denotes the time step in the simulation and the second term in the right-hand side of Eq. (9) is introduced for the stabilization of the numerical computation.

The numerical code for solving the Einstein equation is slightly modified from the previous version as follows: (i) All the spatial finite differencing including the advection term such as $\beta^{i} \partial_{i} \phi$ are evaluated with the fourth-order finite differencing scheme. For the advection term, an upwind method is adopted as proposed, e.g., in [19]. (ii) For the time-integration, a third-order Runge-Kutta scheme is employed. With this scheme, the stable evolution is feasible. Furthermore, the numerical accuracy is signigicantly improved, and as a result, the convergent results can be obtained by a relatively wider grid spacing than by that adopted in the previous papers [15].
The location and properties of the $\mathrm{BH}$ such as the area and circumferential radii are determined using an apparent horizon finder, for which our method is described in [39].

For extracting gravitational waves from the geometrical variables, the gauge-invariant Moncrief variables $R_{l m}$ in the flat spacetime [36] has been computed in our series of papers (e.g., [16, 17, 37]). From $R_{l m}$, the plus and cross modes of gravitational waves, $h_{+}$and $h_{\times}$, are obtained by a simple algebraic calculation. The detailed equations are described in [16, 37] to which the reader may refer. In this method, we split the metric in the wave zone into the flat background and linear perturbation. Then, the linear part is decomposed using the tensor spherical harmonics, and the gauge-invariant variables are constructed for each mode of the eigen values $(l, m)$. The gauge-invariant variables of $l \geq 2$ can be regarded as gravitational waves in the wave zone, and hence, we derive such modes in the numerical simulation.

In the present work, we also compute the outgoing part of the Newman-Penrose quantity (the so-called $\psi_{4}$; see, e.g., [19, 30] for definition of $\left.\psi_{4}\right)$. By twice time integration of $2 \psi_{4}$ (and with an appropriate choice of the integration constants), one can compute the gravitational waveforms. We compare these gravitational waveforms with those computed from the gauge-invariant waveextraction method. It is found that the wave phases of both waveforms agree well throughout the inspiral phase to the tidal disruption phase. However, the amplitude does not agree well in the early inspiral state; the magnitude of the disagreement is $\sim 20-30 \%$, although the disagreement is much smaller during the tidal disruption. The reason is that we extract gravitational waves near the outer boundary which is located in the local wave zone in the present work, and the amplitude is not the correct asymptotic amplitude. To obtain the asymptotic amplitude, it is necessary to perform the extrapolation using the data of different extraction radii. One point worthy to note is that the convergence of the amplitude of $D \psi_{4}$ with changing the extraction radius $D$ is much faster than that for $D R_{l m}$. This indicates that $\psi_{4}$ is a better tool than $R_{l m}$ for evaluating the asymptotic waveforms with smaller systematic error, in the simulation that the outer boundary is located in the local wave zone. Hence, we decide that in this paper, we evaluate the waveform, energy, angular momentum, and linear momentum fluxes analyzing $\psi_{4}$.

We compute the modes of $2 \leq l \leq 4$ for $\psi_{4}$, and found that the mode of $(l,|m|)=(2,2)$ is dominant, but $l=$ $|m|=3$ and $l=|m|=4$ modes also contribute to the energy and angular momentum dissipation by more than $1 \%$ for the merger of the chosen initial data.

We also estimate the kick velocity from the linear momentum flux of gravitational waves. The linear momentum flux $d P_{i} / d t$ is computed from the same method as that given in [19, 38]. Specifically, the coupling terms between $l=|m|=2$ and $l=|m|=3$ modes and between $l=|m|=2$ and $(l,|m|)=(2,1)$ modes primarily con- 
TABLE II: Parameters for the grid in the numerical simulation. The grid number for covering one positive direction $N$, the grid number for the inner uniform-grid domain $N_{0}$, the parameters for the non-uniform grid domain $(\Delta i, \xi)$, the grid spacing in the uniform domain in units of $M_{\mathrm{p}}$, the grid number covered for the major diameter of the NS $\left(L_{\mathrm{NS}}\right)$, and the ratio of the location of the outer boundary along each axis to the gravitational wavelength at $t=0$.

\begin{tabular}{cccccccc}
\hline Model & $N$ & $N_{0}$ & $\Delta i$ & $\xi$ & $\Delta x / M_{\mathrm{p}}$ & $L_{\mathrm{NS}} / \Delta x$ & $L / \lambda$ \\
\hline $\mathrm{A}$ & 225 & 150 & 30 & 20.5 & $1 / 15$ & 55 & 0.86 \\
\hline $\mathrm{B}$ & 225 & 150 & 30 & 20.5 & $1 / 15$ & 49 & 0.86 \\
\hline $\mathrm{C}$ & 225 & 151 & 30 & 20.5 & $1 / 15$ & 63 & 0.86 \\
\hline $\mathrm{D}$ & 225 & 155 & 30 & 20.5 & $1 / 15$ & 67 & 0.74 \\
\hline $\mathrm{E}$ & 225 & 150 & 30 & 20.5 & $1 / 15$ & 59 & 0.82 \\
\hline $\mathrm{F}$ & 225 & 155 & 30 & 22.0 & $1 / 16$ & 50 & 0.80 \\
\hline \hline $\mathrm{A} 1$ & 211 & 136 & 30 & 18.35 & $1 / 13.5$ & 49 & 0.86 \\
\hline $\mathrm{A} 2$ & 195 & 121 & 30 & 16.4 & $1 / 12$ & 44 & 0.86 \\
\hline $\mathrm{C} 2$ & 195 & 121 & 30 & 16.2 & $1 / 12$ & 50 & 0.86 \\
\hline
\end{tabular}

tribute the linear momentum flux. From the total linear momentum dissipated by gravitational waves,

$$
\Delta P_{i}=\int \frac{d P_{i}}{d t} d t
$$

the kick velocity is defined by $\Delta P_{i} / M$ where $M$ is the initial ADM mass of the system.

\section{NUMERICAL RESULTS}

\section{A. Choosing the grid points}

In the simulation, the cell-centered Cartesian, $(x, y, z)$, grid is adopted to avoid the situation that the location of the puncture (which always stays in the $z=0$ plane) coincides with one of the grid points. The plane symmetry is assumed with respect to the equatorial plane. The computational domain of $-L \leq x \leq L,-L \leq y \leq L$, and $0<z \leq L$ is covered by the grid size of $(2 N, 2 N, N)$ for $x-y-z$ where $L$ and $N$ are constants. Following [15, 35], we adopt a nonuniform grid as follows; an inner domain of $\left(2 N_{0}, 2 N_{0}, N_{0}\right)$ grid zone is covered with a uniform grid of the spacing $\Delta x$ and outside this inner domain, the grid spacing is increased according to the relation of $\xi \tanh \left[\left(i-N_{0}\right) / \Delta i\right] \Delta x$ where $i$ denotes the $i$-th grid point in each positive direction, and $N_{0}, \Delta i$, and $\xi$ are constants. Then, the location of the $i$-th grid, $x^{k}(i)$, in each direction is

$$
x^{k}(i)= \begin{cases}(i-1 / 2) \Delta x & 1 \leq i \leq N_{0} \\ (i-1 / 2) \Delta x+\xi \Delta i \Delta x & \\ \times \log \left[\cosh \left\{\left(i-N_{0}\right) / \Delta i\right\}\right] & i>N_{0}\end{cases}
$$

and $x^{k}(-i)=-x^{k}(i) \quad i=1,2, \cdots, N$ for $x^{k}=x$ and $y$.
For all the simulations, we chose $N=225$ and $\Delta i=30$. For most of the simulation, we determined the grid spacing for the inner uniform domain according to the rule $\Delta x / M_{\mathrm{p}}=1 / 15$ where $M_{\mathrm{p}}$ is the mass parameter of the puncture. We have found that with this choice of the grid size, a convergent result for the $\mathrm{BH}$ orbit is obtained. Actually, the simulations for the BH-BH binary in the moving puncture framework (e.g., 18, 19, 31]) show that this choice is appropriate for obtaining a convergent result in the fourth-order scheme. With increasing the mass ratio $M_{\mathrm{p}} / M_{\mathrm{NS}}$, however, the grid number of covering the NS becomes small for the fixed value of $\Delta x$. We require that the major diameter of the NS, $L_{\mathrm{NS}}$, should be covered by $\gtrsim 50$ grid points, because a result with small error is obtained with such setting (see Appendix A). By this reason, for model $\mathrm{F}$, we choose $\Delta x / M_{\mathrm{p}}=1 / 16$.

In Table II, we list the parameters for the grid coordinates. $\lambda$ denotes the wavelength of gravitational waves at $t=0(\lambda=\pi / \Omega) . \quad N_{0}$ is chosen so as to put the NS in the inner uniform-grid domain initially. $\xi$ is determined from the conditions (i) $L / \lambda \gtrsim 0.75$ and (ii) the grid spacing near the outer boundaries is smaller than $1.05 \mathrm{M}$. Note that $\lambda$ decreases with the time because the orbital radius decreases due to the gravitational radiation reaction. Hence, $L$ is smaller than $\lambda$ at the onset of tidal disruption. The gravitational wavelength after the merger sets in is expected to be larger than $11 M$ which is approximately equal to the wavelength of the quasinormal mode $(\mathrm{QNM})$ of the $\mathrm{BH}$ excited in the final phase of the merger. Because the wavelength is covered by at least 10 grid points, we may expect that gravitational waves are extracted with a good accuracy near the outer boundary.

To see the dependence of the numerical results on the grid resolution, we performed test simulations for $\Delta x=$ $M_{\mathrm{p}} / 12$ (models A2 and $\mathrm{C} 2$ ), and for $\Delta x=M_{\mathrm{p}} / 13.5$ (model A1). For models A, A1, and A2 and for models $\mathrm{C}$ and $\mathrm{C} 2$, the locations of the outer boundaries are approximately the same, respectively (see Table II). We found that the numerical results (see Appendix A) show an acceptable convergence for the case that the grid spacing satisfies the conditions (i) $\Delta x \geq M_{\mathrm{p}} / 12$ and (ii) that the diameter of the NS is covered by more than $\approx 50$ grid points. As we discuss in Appendix A, the errors for the estimate of the mass surrounding the $\mathrm{BH}$ and area of the apparent horizon at the end of the simulation are within $\sim 10 \%-30 \%\left(\sim 0.01-0.02 M_{\odot}\right)$ and $\sim 0.3 \%-0.5 \%$, respectively, for such setting. The total energy and angular momentum emitted by gravitational waves are also computed within $\sim 10 \%-20 \%$ error. In the previous papers 15], we performed simulations in the second-order accurate finite-differencing for solving the Einstein equation. In such case, the convergence of the numerical results with improving the grid resolution is not very fast. In the present work, we employ the fourth-order scheme, with which the convergence is achieved much faster. 

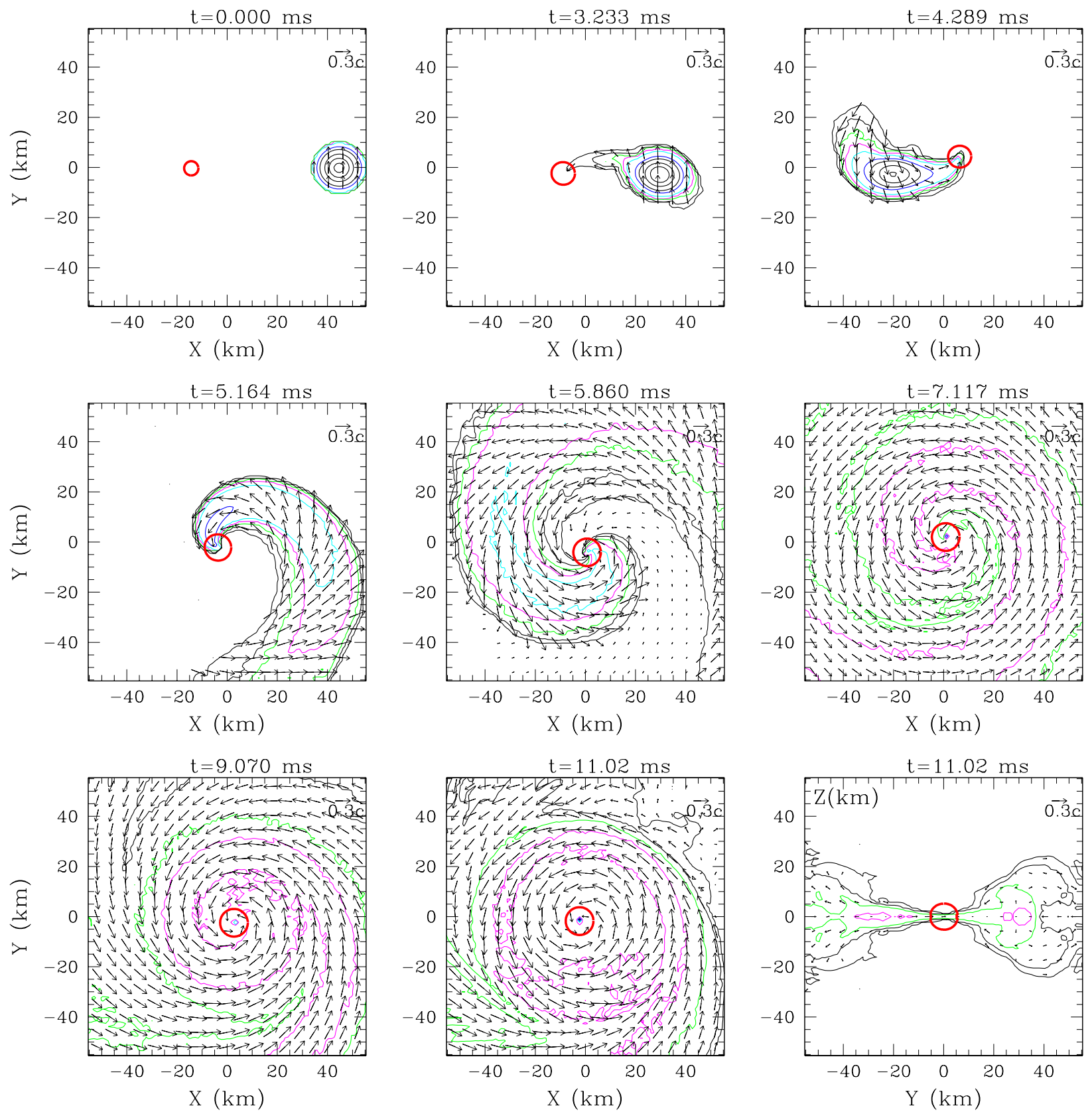

FIG. 1: Snapshots of the density contour curves for $\rho$ and the three velocity for $v^{i}\left(=d x^{i} / d t\right)$ in the equatorial plane for model A. The solid contour curves are drawn for $\rho=2 i \times 10^{14} \mathrm{~g} / \mathrm{cm}^{3}(i=1-4)$ and for $10^{14-i} \mathrm{~g} / \mathrm{cm}^{3}(i=1 \sim 5)$. The maximum density at $t=0$ is $\approx 8.86 \times 10^{14} \mathrm{~g} / \mathrm{cm}^{3}$. The blue, cyan, magenta, and green curves denote $10^{14}, 10^{13}, 10^{12}$, and $10^{11} \mathrm{~g} / \mathrm{cm}^{3}$, respectively. The vectors shows the velocity field $\left(v^{x}, v^{y}\right)$, and the scale is shown in the upper right-hand corner. The thick (red) circles are the apparent horizons. The 2nd and 3rd panels denote the states after one and one-and-half orbits, respectively. The last panel plots the density contours and velocity vectors in the $x=0$ plane at the same time as that for the 8 th panel. 


\section{B. Atmosphere}

Because any conservation scheme of hydrodynamics is unable to evolve a vacuum, we have to introduce an artificial atmosphere outside the NS. However, if the density of the atmosphere was too large, it might affect the orbital motions of the $\mathrm{BH}$ and NS and moreover we might overestimate the total amount of the rest mass of the formed torus surrounding a BH. Thus, we initially assign a small rest-mass density as follows:

$$
\rho= \begin{cases}\rho_{\mathrm{at}} & r \leq x\left(N_{0}\right) \\ \rho_{\mathrm{at}} e^{1-r / x\left(N_{0}\right)} & r>x\left(N_{0}\right) .\end{cases}
$$

Here, we choose $\rho_{\text {at }}=\rho_{\max } \times 10^{-8}$ where $\rho_{\max }$ is the maximum rest-mass density of the NS. With such choice, the total amount of the rest mass of the atmosphere is about $10^{-5}$ of the rest mass of the NS. Thus, the accretion of the atmosphere onto the NS and $\mathrm{BH}$ plays a negligible role for their orbital evolution in the present context. In the following, we discuss the rest mass of the torus surrounding a $\mathrm{BH}$. As we show below, the rest mass is larger than $0.01 M_{\odot}$ which is much larger than the atmosphere mass. Hence, the atmosphere also plays a negligible role for determining the properties of the torus.

\section{General process of tidal disruption}

All the numerical simulations were performed from about one and half orbits before the onset of tidal disruption to the time in which the accretion time scale of the rest mass of material into the $\mathrm{BH}$ is much longer than the dynamical time scale. The duration of the simulations is 400-500M in units of the ADM mass. In this section, we first describe the general process of tidal disruption, subsequent evolution of the $\mathrm{BH}$, and formation of the accretion disk (torus) showing the numerical results for model A.

Figure 1 plots the evolution of the contour curves for $\rho$ and the velocity vectors for the three velocity $v^{i}\left(=d x^{i} / d t\right)$ in the equatorial plane for model $\mathrm{A}$. The location of the apparent horizons is shown together. Due to gravitational radiation reaction, the orbital radius decreases and then the NS is elongated gradually (panels 2 and 3). The tidal disruption of the NS by the $\mathrm{BH}$ sets in at $t \sim 4.3 \mathrm{~ms}$ (at about one and half orbits; panel 3). Soon after this time, the outer part of the NS expands outward due to angular momentum transport by the hydrodynamic interaction. This material subsequently forms a torus around the BH. However, the tidal disruption sets in at an orbit very close to the ISCO and hence the material in the inner part is quickly swallowed into the $\mathrm{BH}$ (4rd and 5th panels). By the outward angular momentum transport, the material in the outer part of the NS forms a one-armed spiral arm (5th and 6th panels). The spiral arm then winds around the $\mathrm{BH}$, and the material, which does not have angular momentum large enough to orbit the $\mathrm{BH}$, shrinks and falls into the $\mathrm{BH}$ (7th panel). A relatively large fraction of the material falls in particular for $t \approx 9-11 \mathrm{~ms}$ (see also the upper panel of Fig. 5(a)). In this phase, the inner and outer parts of the spiral arm collide and thermal energy is generated due to the shock heating. The high-density part of the spiral arm then expands to form a compact torus with the maximum density $\sim 10^{12} \mathrm{~g} / \mathrm{cm}^{3}$ (8th and 9th panels; see also Fig. 21). We stopped the simulation at $t \approx 12.5 \mathrm{~ms}$, at which the rest mass of the material located outside the apparent horizon is $\approx 0.092 M_{\odot}$.

We define the specific thermal energy generated by the shocks by

$$
\varepsilon_{\mathrm{th}} \equiv \varepsilon-\frac{\kappa}{\Gamma-1} \rho^{\Gamma}
$$

where the second term in the right-hand side is the polytropic term, i.e., the specific internal energy in the absence of the shocks. $\varepsilon_{\text {th }}$ is zero in the adiabatic evolution (in the absence of the shocks), and hence, $\varepsilon_{\text {th }}$ may be regarded as the specific thermal energy generated by the shocks. Figure 2 (top panel of each) plots $\varepsilon_{\text {th }}$ along $x$ and $y$ axes at $t=7.117,9.070$, and $11.02 \mathrm{~ms}$. The ratio of the thermal energy to the total internal energy $\varepsilon_{\mathrm{th}} / \varepsilon$ and the rest-mass density are shown together. This figure shows that the generated thermal energy is much larger than the polytropic one for most region except for the high-density region of the spiral arms which do not experience the shock heating. However, during the evolution, the spiral arms winds around the $\mathrm{BH}$ and the shocked region increases. As a result, $\varepsilon_{\mathrm{th}} / \varepsilon$ eventually becomes approximately equal to unity for all the regions. Figure 2 indicates that a hot torus with the density $\sim 10^{11}$ $10^{12} \mathrm{~g} / \mathrm{cm}^{3}$ is the final outcome. This is a favorable property for producing a large amount of neutrinos which may drive SGRBs (e.g., [40, 41, 42, 43]).

Although the EOS adopted in this paper is idealized one, we approximately estimate the temperature of the torus. Assuming that the specific thermal energy is composed of those of the gas, photons, and relativistic electrons (and positrons), we have [42, 43]

$$
\varepsilon_{\mathrm{th}}=\frac{3 k T}{2 m}+\frac{11 a_{r} T^{4}}{\rho},
$$

where $k, a_{r}, T$, and $m$ are the Boltzmann constant, radiation-density constant, temperature, and mass of the main component of nucleons. For simplicity, we assume that the gas is composed of the neutron and set $m=1.66 \times 10^{-24} \mathrm{~g}$. Because the temperature is higher than $\sim 6 \times 10^{9} \mathrm{~K}$ (see below), we assume that the electrons and positrons are relativistic to describe Eq. (14). If the density is high, the neutrinos are optically thick and contribute to the thermal energy. Although this effect is important for $\rho \gtrsim 10^{12} \mathrm{~g} / \mathrm{cm}^{3}$ [40, 41, 42, 43], the density of the outcome is at most $10^{12} \mathrm{~g} / \mathrm{cm}^{3}$, and hence, we ignore it. Then, the radiation energy is smaller than the gas energy for $\rho \gtrsim 10^{11} \mathrm{~g} / \mathrm{cm}^{3}$ and $\varepsilon_{\mathrm{th}} / c^{2} \lesssim 0.01$. 

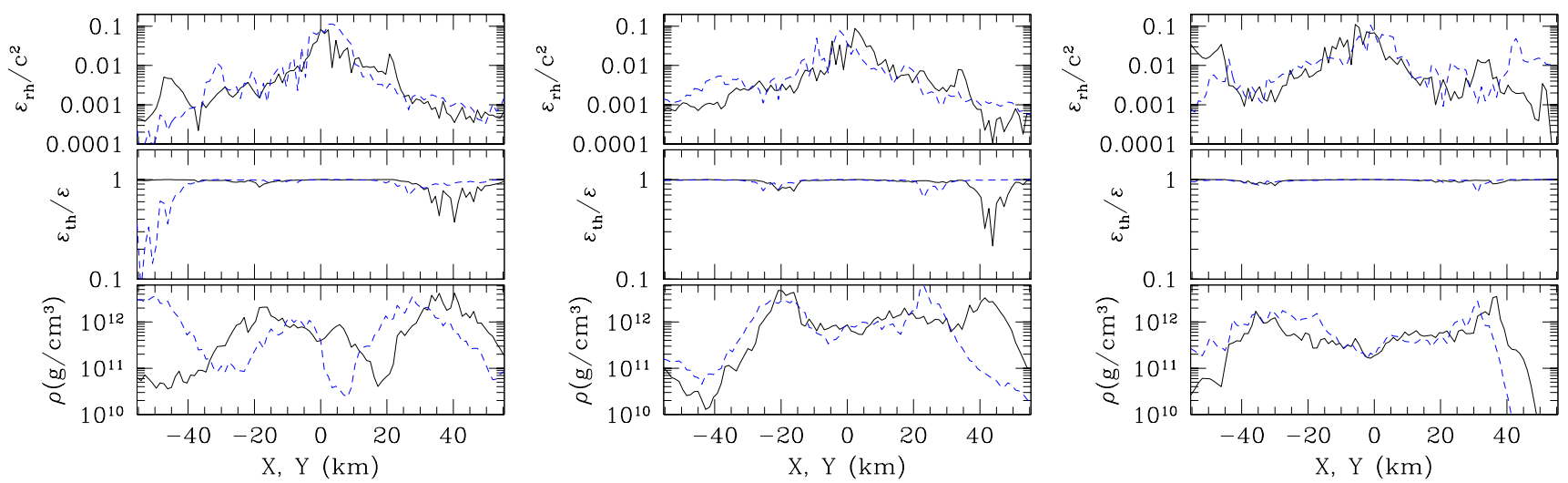

FIG. 2: Profiles of $\varepsilon_{\mathrm{th}}, \varepsilon_{\mathrm{th}} / \varepsilon$, and $\rho$ along $x$-axis (solid curves) and $y$-axis (dashed curves) at $t=7.117 \mathrm{~ms}$ (left), $9.070 \mathrm{~ms}$ (middle), and 11.02 (right).
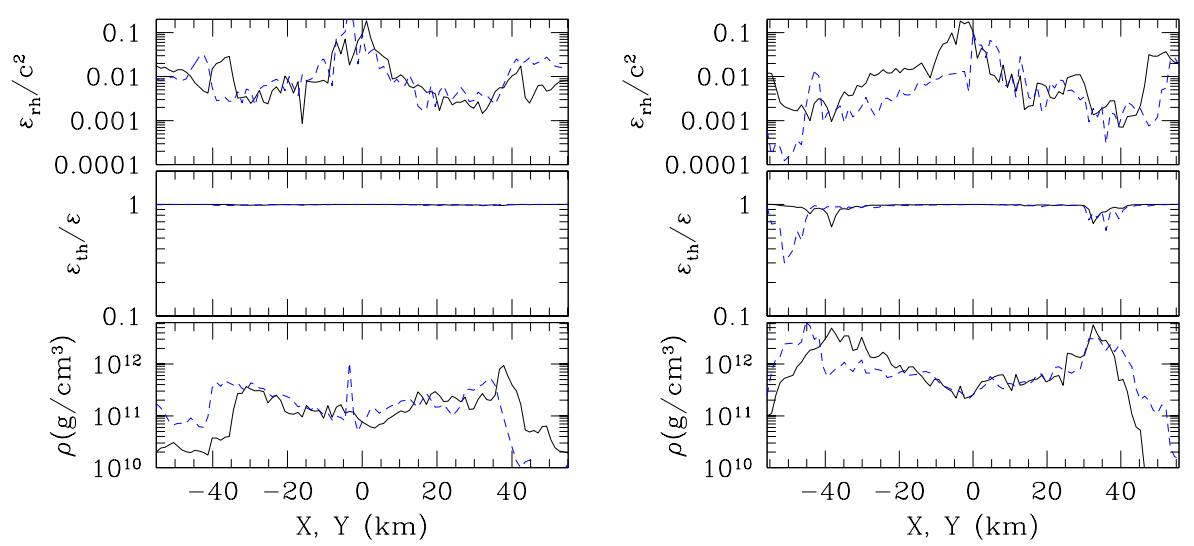

FIG. 3: Profiles of $\varepsilon_{\mathrm{th}}, \varepsilon_{\mathrm{th}} / \varepsilon$, and $\rho$ along $x$-axis (solid curves) and $y$-axis (dashed curves) at $t=11.02$ for models B (left) and C (right).

From Eq. (14), the temperature is approximately given by

$$
T \approx 7.2 \times 10^{10}\left(\frac{\varepsilon_{\mathrm{th}}}{0.01}\right) \mathrm{K} .
$$

Thus, the estimated temperature is between $10^{10}$ and $\sim 10^{11} \mathrm{~K}$ for the torus. (Note that $\varepsilon \sim 0.1$ inside the apparent horizon, but we do not pay attention to the inside of the $\mathrm{BH}$.)

For clarification of the dependence of the density and the thermal energy on the radius of the tidally disrupted NSs, $R_{\mathrm{NS}}$, we show the same plots as Fig. 2 for models B and $\mathrm{C}$ in Fig. 3. We also plot the density contour curves and velocity vectors at $t=11.02 \mathrm{~ms}$ for these models in Fig. 4. Figure 3 shows that the value of $\varepsilon_{\mathrm{th}}$ increases above 0.001 dominating the specific thermal energy irrespective of models. Figure 4 shows that irrespective of the models, the outcome after the tidal disruption is a compact torus surrounding the BH. Thus, the hot torus is the universal outcome irrespective of $R_{\mathrm{NS}}$ as long as it is in the range between 12 and $15 \mathrm{~km}$.
By contrast, the rest-mass density depends sensitively on the torus mass (see Table III and next section for the final values of the rest mass of the material located outside the apparent horizon which is approximately equal to the torus mass). For model B, the torus mass is much smaller than that for model A. This is simply because the NS radius is smaller and hence the tidal disruption sets in at an orbit very close to the ISCO. For model $\mathrm{C}$, by contrast, the torus mass is much larger than those for models A and B because of the larger NS radius. The averaged rest-mass density has a clear correlation with the torus mass. For model B, the density is smaller than $10^{12} \mathrm{~g} / \mathrm{cm}^{3}$ for most region of the torus, whereas for model C, the density is larger than $10^{12} \mathrm{~g} / \mathrm{cm}^{3}$ for most region. For such high-density case, the neutrinos are likely to be optically thick [40], and the neutrinodominated accretion flow will be subsequently formed. 

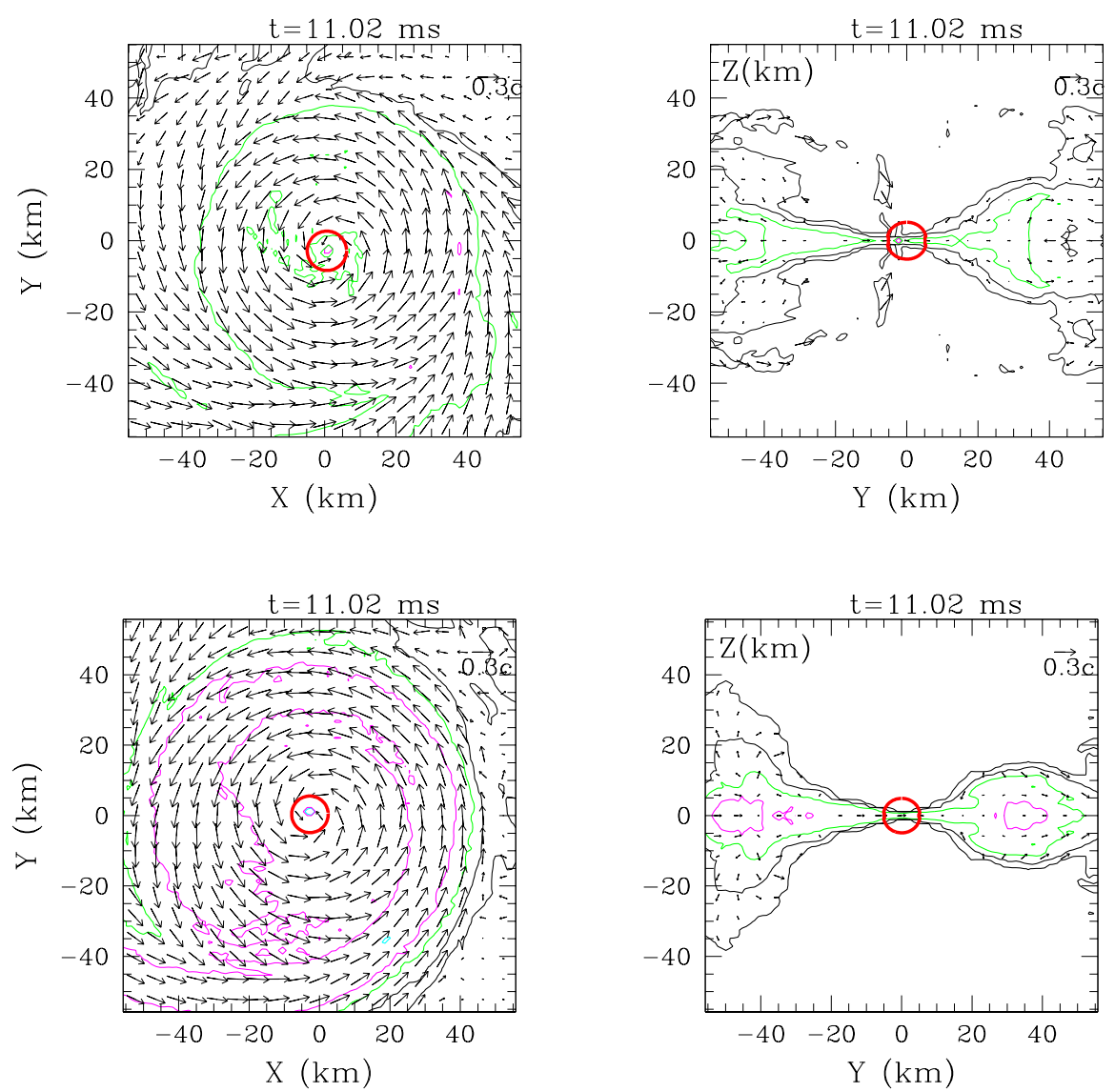

FIG. 4: The same as Fig. 1 but for models B and C at $t=11.02 \mathrm{~ms}$. The upper two panels plot the contour plots and velocity vectors for $x-y$ and $y-z$ planes for model B. The lower two panels show the plots for model C.

\section{Torus mass}

The upper panels of Fig. [5(a)-(c) plot the evolution of the fraction of the rest mass of the material located outside the apparent horizon $M_{r>r_{\mathrm{AH}}}$ (a) for models A, $\mathrm{D}$, and F, (b) for models B and E, and (c) for models A-C. Here, $M_{r>r_{\mathrm{AH}}}$ is defined by

$$
M_{r>r_{\mathrm{AH}}} \equiv \int_{r>r_{\mathrm{AH}}} \rho \alpha u^{t} \sqrt{\gamma} d^{3} x
$$

and $u^{t}$ is the time component of the four-velocity. We find that a large fraction of the material is swallowed into the $\mathrm{BH}$ soon after the onset of merger irrespective of the models (see also Table III). However, the final value of $M_{r>r_{\mathrm{AH}}}$ depends strongly on the NS radius; e.g., for $R_{\mathrm{NS}}=14.7 \mathrm{~km}$ and $q \approx 0.33$ (model $\mathrm{C}$ ), $\approx 12 \%$ of the material is located outside the $\mathrm{BH}$ at the end of the simulation, whereas for $R_{\mathrm{NS}}=12.0 \mathrm{~km}$ and $q \approx 0.33$ (model $\mathrm{B}$ ), only $\approx 2 \%$ of the material is located outside the $\mathrm{BH}$ at the end of the simulation (see Fig. 5(c)). The final value of $M_{r>r_{\mathrm{AH}}}$ depends also on the mass ratio $q$. Figure 5(a) illustrates that the final value of $M_{r>r_{\mathrm{AH}}}$ increases by $\sim 75 \%$ for the increase of the value of $q$ from 0.28 to 0.39. Figure 5(b) also illustrates that the final value of
$M_{r>r_{\mathrm{AH}}}$ increases by $\sim 50 \%$ for the small increase of $q$ from 0.33 to 0.39 .

Figure 5(b) and (c) (in particular (c)) clarifies the dependence of the infall process of the tidally disrupted material into the $\mathrm{BH}$ on the NS radius. With the decrease of the NS radii, the accretion rate of the rest mass into the $\mathrm{BH}$ at the onset of tidal disruption increases steeply. Furthermore, the accretion time scale shortens; e.g., for model $\mathrm{B}$, the duration of the quick accretion is shorter than $1 \mathrm{~ms}$, whereas for model $\mathrm{C}$, it is $\sim 1.5 \mathrm{~ms}$.

Figure 5(d) plots the values of $M_{r>r_{\mathrm{AH}}}$ at the end of the simulations as a function of $R_{\mathrm{NS}}$ for given values of the mass ratio $q$. It is clearly seen that the fraction of the material located outside the BH steeply decreases with the decrease of the NS radius. Extrapolating the results for the smaller values of $R_{\mathrm{NS}}$, we expect that $M_{r>r_{\mathrm{AH}}}$ would become close to zero for $R_{\mathrm{NS}} \lesssim 11 \mathrm{~km}$ both for $q=0.33$ and 0.39 . For such case, a very small value of the BH mass as $M_{\mathrm{BH}}<3 M_{\odot}$ would be required for the formation of torus of mass $\gtrsim 0.01 M_{\odot}$. 

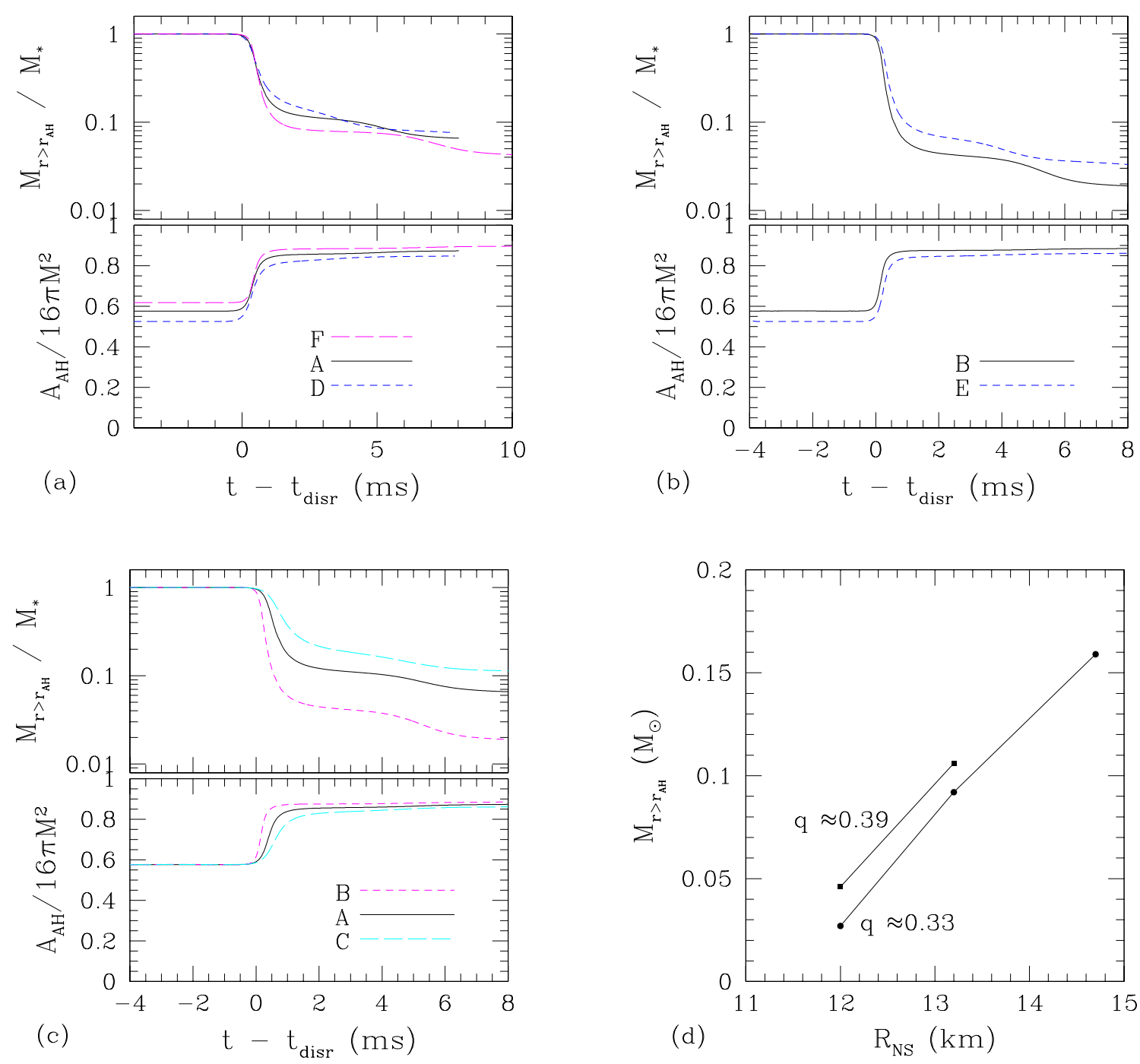

FIG. 5: The evolution of the rest mass of the material located outside the apparent horizon (upper panel of each figure) and the evolution of the area of the apparent horizon in units of $16 \pi M^{2}$ (lower panel of each figure) (a) for models $\mathrm{A}$, D, and $\mathrm{F}$ (for $R_{\mathrm{NS}}=13.2 \mathrm{~km}$ ), (b) for models $\mathrm{B}$ and $\mathrm{E}$ (for $R_{\mathrm{NS}}=12 \mathrm{~km}$ ), and (c) for models $\mathrm{A}, \mathrm{B}$, and $\mathrm{C}$ (for $q \approx 0.33$ ). $t_{\mathrm{disr}}$ in the horizontal axis denotes the approximate time at which the tidal disruption sets in, and we determine it to be 4.5 ms for model A, $4.2 \mathrm{~ms}$ for mode B, $4.3 \mathrm{~ms}$ for mode C, $4.2 \mathrm{~ms}$ for model D, $3.9 \mathrm{~ms}$ for model E, and $5.6 \mathrm{~ms}$ for model F, respectively. (d) $M_{r>r_{\mathrm{AH}}}$ at the end of the simulations as a function of $R_{\mathrm{NS}}$.

\section{E. Black hole mass and spin}

The lower panels of Fig. [5(a)-(c) plot the evolution of the area of the apparent horizon in units of $16 \pi M^{2}$. The area of the BHs is constant before the onset of tidal disruption. The value of the area fluctuates only by $\sim 0.03 \%$ in such phase. After the onset of tidal disruption, the area quickly increases as a result of the mass accretion, and finally, approaches an approximate constant. From the final values of the area of the apparent horizon together with the estimated $\mathrm{BH}$ mass at the final stage, $M_{\mathrm{BH}, \mathrm{f}}$, the nondimensional spin parameter of the formed
$\mathrm{BHs}, a$, is approximately derived from

$$
\hat{A} \equiv \frac{A}{16 \pi M_{\mathrm{BH}, \mathrm{f}}^{2}}=\frac{1+\sqrt{1-a^{2}}}{2} .
$$

To approximately estimate $M_{\mathrm{BH}, \mathrm{f}}$, we use the following equation as in 15]

$$
M_{\mathrm{BH}, \mathrm{f}} \equiv M-M_{r>r_{\mathrm{AH}}}-E_{\mathrm{GW}},
$$

where $E_{\mathrm{GW}}$ is the total radiated energy by gravitational waves 52]. The results for $E_{\mathrm{GW}}, M_{r>r_{\mathrm{AH}}}, M_{\mathrm{BH}, \mathrm{f}}$, and $\hat{A}$ are listed in Table III. The gravitational wave energy is primarily carried by the $l=|m|=2$ modes. We found that $l=|m|=3$ and $l=|m|=4$ modes are subdominant 
TABLE III: The total radiated energy in units of the initial ADM mass $M$, the total radiated angular momentum in units of the initial angular momentum $J$, the kick velocity, the rest mass of the material located outside the apparent horizon, the estimated $\mathrm{BH}$ mass, the area of the apparent horizon in units of $16 \pi M_{\mathrm{BH}, \mathrm{f}}^{2}$, the ratio of the polar circumferential radius to the equatorial one of the apparent horizon $\left(C_{p} / C_{e}\right)$, and the estimated spin parameters of the final state of the BH. $a_{\mathrm{f} 1}, a_{\mathrm{f} 2}$, and $a_{\mathrm{f} 3}$ are computed from the area of the apparent horizon, the estimated angular momentum and mass of the $\mathrm{BH}$, and $C_{p} / C_{e}$, respectively. All the values presented here are measured for the states obtained at the end of the simulations. We note that $C_{p} / C_{e}$ varies with time by $\approx 0.05 \%$. The energy, angular momentum, and linear momentum fluxes of gravitational waves are evaluated changing the location of the extraction, and we found that the energy and angular momentum fluxes converge within $\sim 1 \%$ error, whereas the error of the linear momentum flux is $\sim 10 \%$.

\begin{tabular}{ccccccccccc}
\hline Model & $\Delta E / M$ & $\Delta J / J$ & $V_{\text {kick }}(\mathrm{km})$ & $M_{r>r_{\mathrm{AH}}}\left(M_{\odot}\right)$ & $M_{\mathrm{BH}, \mathrm{f}}\left(M_{\odot}\right)$ & $\hat{A}_{\mathrm{AH}}$ & $C_{p} / C_{e}$ & $a_{\mathrm{f} 1}$ & $a_{\mathrm{f} 2}$ & $a_{\mathrm{f} 3}$ \\
\hline $\mathrm{A}$ & $0.68 \%$ & $11.3 \%$ & 19 & 0.092 & 5.099 & 0.9175 & 0.9391 & 0.550 & 0.560 & 0.545 \\
\hline $\mathrm{B}$ & $0.96 \%$ & $12.9 \%$ & 13 & 0.027 & 5.116 & 0.9110 & 0.9350 & 0.570 & 0.577 & 0.561 \\
\hline $\mathrm{C}$ & $0.45 \%$ & $8.7 \%$ & 18 & 0.159 & 5.078 & 0.9241 & 0.9437 & 0.530 & 0.543 & 0.526 \\
\hline $\mathrm{D}$ & $0.61 \%$ & $10.3 \%$ & 17 & 0.106 & 4.442 & 0.8994 & 0.9260 & 0.597 & 0.612 & 0.595 \\
\hline $\mathrm{E}$ & $0.93 \%$ & $13.0 \%$ & 19 & 0.046 & 4.458 & 0.8953 & 0.9220 & 0.612 & 0.622 & 0.608 \\
\hline $\mathrm{F}$ & $0.77 \%$ & $12.5 \%$ & 4 & 0.060 & 5.773 & 0.9288 & 0.9477 & 0.514 & 0.525 & 0.508 \\
\hline
\end{tabular}

modes; for $q \approx 0.33(0.39)$, the total emitted energies carried by $l=|m|=3$ and $l=|m|=4$ modes are $\sim 5$ $6 \%(4 \%)$ and $\sim 1-2 \%$ (1\%) of that of $l=|m|=2$ modes, respectively.

The nondimensional spin parameter determined from $\hat{A}$ is listed in Table III as $a_{\mathrm{f} 1}$. We find that $a_{\mathrm{f} 1}$ is between $\sim 0.5$ and $\sim 0.6$ for all the models, implying that rotating BHs of moderate magnitude of the spin are the final outcomes. For the larger mass ratio $q$, the spin parameter is larger for the same NS radius. The reason for this is that for the larger mass ratio, the initial total angular momentum of the system $J$ is larger (see Table I). For the smaller NS radius, the spin parameter is larger for the same mass ratio. This is simply because the larger fraction of the material falls into the $\mathrm{BH}$ resulting in the spin up.

To check the validity of the above estimate, we evaluate the nondimensional spin parameter employing other two methods by which the BH spin may be approximately calculated. In the first method, the spin is evaluated directly from the angular momentum of the $\mathrm{BH}$ defined by

$$
J_{\mathrm{BH}, \mathrm{f}} \equiv J-J_{r>r_{\mathrm{AH}}}-J_{\mathrm{GW}},
$$

where $J_{\mathrm{GW}}$ is the total radiated angular momentum by gravitational waves and $J_{r>r_{\mathrm{AH}}}$ is the angular momentum of the material located outside the apparent horizon, which is defined by

$$
J_{r>r_{\mathrm{AH}}} \equiv \int_{r>r_{\mathrm{AH}}} \rho \alpha h u^{t} u_{\varphi} \sqrt{\gamma} d^{3} x .
$$

Here, $u_{\varphi}$ is the $\varphi$ component of the four-velocity, and $h$ is the specific enthalpy defined by $1+\varepsilon+P / \rho$. $J_{\mathrm{BH}, \mathrm{f}}$ exactly gives the angular momentum of the material in the axisymmetric and stationary spacetime. In the late phase of the merger, the spacetime relaxes to a quasistationary and nearly axisymmetric state. Thus, we may expect that $J_{r>r_{\mathrm{AH}}}$ will provide an approximate magnitude of the angular momentum of the torus.
From $J_{\mathrm{BH}, \mathrm{f}}$ and $M_{\mathrm{BH}, \mathrm{f}}$, we define the nondimensional spin parameter by $a_{\mathrm{f} 2} \equiv J_{\mathrm{BH}, \mathrm{f}} / M_{\mathrm{BH}, \mathrm{f}}^{2}$ (see Table III). It is found that $a_{\mathrm{f} 2}$ is systematically larger than $a_{\mathrm{f} 1}$ but these two values, independently determined, still agree within $\approx 2.5 \%$ disagreement. This suggests that both quantities denote the BH spin within the error of $\sim 2.5 \%(\sim 0.015)$. We note that because the relation $a_{\mathrm{f} 1}<a_{\mathrm{f} 2}$ holds systematically, this disagreement would primarily result from the systematic error associated with the approximate definitions of the spin. For example, we use the rest mass $M_{r>r_{\mathrm{AH}}}$ to estimate the disk mass in Eq. (18), ignoring the gravitational binding energy between the $\mathrm{BH}$ and the torus. Also, Eq. (20) may include an error because the torus is neither exactly axisymmetric nor stationary.

In the second method, we use the ratio of the polar circumferential length to the equatorial one of the $\mathrm{BH}$, $C_{p} / C_{e}$. We compare this ratio to that of the Kerr $\mathrm{BH}$, and estimate the spin parameter. The results are listed in Table III as $a_{\mathrm{f} 3}$. Again we find that this agrees with other two with a good accuracy (within $\sim 1.5 \%$ disagreement with $\left.a_{\mathrm{f} 1}\right)$. We note that the axial ratio varies with time by $\sim 0.05 \%$. This implies that the error for the estimation of $a_{\mathrm{f} 3}$ is $\sim 0.5 \%$ for $a_{\mathrm{f} 3}=0.5-0.6$. We also note that the relation between $C_{p} / C_{e}$ and the spin parameter in the presence of the torus is slightly different from that of the Kerr BH [44]. However, the systematic error is negligible because the torus mass is only $\lesssim 3 \%$ of the $\mathrm{BH}$ mass.

From these results, we conclude that the spin parameter obtained by three different methods agree within $\sim 2.5 \%$ error. We note that all three methods can only approximately determine the spin, and the systematic error associated with their definition should be included. Actually, the systematic relation $a_{\mathrm{f} 2}>a_{\mathrm{f} 1}>a_{\mathrm{f} 3}$ holds. Hence, the error of $\sim 2.5 \%$ results not only from the numerical error but also from the systematic error.

The spin parameter of the formed BHs is smaller than the initial value of $J / M^{2}$ of the system by $14-20 \%$. The primary reason for this in the case that most of the NS matter falls into the $\mathrm{BH}$, i.e., for $R_{\mathrm{NS}}=12 \mathrm{~km}$, is that 
gravitational waves carry away the angular momentum by $\sim 13 \%$ of $J$ (see Table II). By contrast, for the case that $M_{r>r_{\mathrm{AH}}}$ is large, the angular momentum of the torus occupies a large fraction of $J$; e.g., for model $\mathrm{C}$, in which $M_{r>r_{\mathrm{AH}}} \approx 0.16 M_{\odot}, J_{r>r_{\mathrm{AH}}}$ is about $15 \%$ of $J$. In such case, the angular momentum of the torus is about twice larger than that carried by gravitational waves.

\section{F. Gravitational waves}

Figure 6(a) and (b) plot gravitational waveforms observed along the $z$-axis as a function of the retarded time for model A. The retarded time is approximately defined by

$$
t_{\mathrm{ret}} \equiv t-D-2 M \ln (D / M),
$$

where $D$ is the distance to the observer. The upper panel of Fig. 6(a) plots + and $\times$ modes of gravitational waves and the lower one the Newman-Penrose quantity. The gravitational waveforms are obtained from twice time integration of the Newman-Penrose quantity. Because the $l=|m|=2$ mode is dominant in the observation along the $z$-axis, we determine the waveforms only from this mode.

From the values of $h_{+}$and $h_{\times}$, the amplitude of gravitational waves at a distance $D$ is evaluated

$$
\begin{aligned}
h_{\mathrm{gw}} \approx 5.0 \times 10^{-22} & \left(\frac{\sqrt{h_{+}^{2}+h_{\times}^{2}}}{0.2}\right) \\
& \times\left(\frac{100 \mathrm{Mpc}}{D}\right)\left(\frac{M_{0}}{5.2 M_{\odot}}\right) .
\end{aligned}
$$

Figure 6(a) implies that the maximum amplitude at a distance of $D=100 \mathrm{Mpc}$ is $\approx 4 \times 10^{-22}$ for model A.

For $t_{\text {ret }} \lesssim 4.5 \mathrm{~ms}$, the inspiral waveforms are seen: The amplitude increases and characteristic wavelength decreases with time. The wavelength at the last inspiral orbit is $\sim 0.8 \mathrm{~ms}$, indicating that the orbital period at the complete tidal disruption is $\sim 1.6 \mathrm{~ms}$, i.e., the angular velocity is $\sim 0.10 M^{-1}$. This value coincides approximately with that of the ISCO, but does not agree with $\Omega$ calculated from Eq. (4) (see Sec. I for discussion). This indicates that the tidal disruption may set in approximately at the orbit predicted by the study of the quasicircular orbits but completes near the ISCO.

For $4.5 \mathrm{~ms} \lesssim t_{\text {ret }} \lesssim 5.5 \mathrm{~ms}$, the amplitude of gravitational waves decreases quickly. In this phase, the tidal disruption and resultant quick accretion of the material into the $\mathrm{BH}$ proceed (see Fig. 5). Thus, gravitational waves could be emitted both by the matter motion and by the quasinormal mode (QNM) oscillation of the $\mathrm{BH}$. The characteristic oscillation period in this phase is $\approx 2.9$ $\mathrm{kHz}$. This value is slightly smaller than the value predicted from the perturbation study for the fundamental QNM of $\mathrm{BH}$ mass $M_{\mathrm{BH}, \mathrm{f}} \approx 5.10 M_{\odot}$ and $\operatorname{spin} a=0.55$.
Here, the perturbation study predicts the frequency and damping time scale as [45]

$$
\begin{aligned}
& \left.f_{\mathrm{qnm}} \approx 3.23\left(\frac{M_{\mathrm{BH}, \mathrm{f}}}{10 M_{\odot}}\right)^{-1}\left[1-0.63(1-a)^{0.3}\right] \mathrm{kH} \& 23\right) \\
& t_{d} \approx \frac{2(1-a)^{-0.45}}{\pi f}
\end{aligned}
$$

which gives $f_{\mathrm{qnm}} \approx 3.19 \mathrm{kHz}$. This indicates that gravitational waves are primarily emitted by the matter motion in this phase; the damping of the amplitude is not due to the QNM damping but to the fact that the compactness and degree of nonaxisymmetry of the matter distribution decrease by the tidal disruption.

For $t \gtrsim 5.5 \mathrm{~ms}$, the rapid accretion stops and the BH approaches a nearly stationary state (see Fig. 5(a)). Hence, for $t_{\text {ret }} \gtrsim 5.5 \mathrm{~ms}$, the ring-down waveforms of the QNM should be seen. Because gravitational waves are also emitted by the matter moving around the $\mathrm{BH}$, the waveforms are modulated and the waveforms associated only by the QNM are not clearly seen. However, $\psi_{4}$ with $5.4 \mathrm{~ms} \lesssim t_{\mathrm{ret}} \lesssim 6 \mathrm{~ms}$ can be fitted by the damping waveforms of the fundamental QNM fairly well. Figure 6(b) plots the $l=|m|=2$ mode of $\psi_{4}$ and a fitted waveform as

$$
A e^{-t / t_{d}} \sin \left(2 \pi f_{\mathrm{qnm}} t+\delta\right),
$$

where $A$ and $\delta$ are constants. For the fitting, we choose the $\mathrm{BH}$ mass and spin as $5.10 M_{\odot}$ and $a=0.55$. In this case, $f_{\mathrm{qnm}}=3.19 \mathrm{kHz}$ and $t_{d}=0.286 \mathrm{~ms}$, respectively [45]. This figure shows that gravitational waves are primarily characterized by the fundamental QNM, and the gravitational wave amplitude of the QNM is much smaller than that at the last inspiral orbit. The possible reason for this small amplitude is that the QNM is excited only incoherently by the infall of the tidally disrupted noncompact material into the $\mathrm{BH}$.

The characteristic feature of the gravitational waveforms emitted after the tidal disruption sets in is that the amplitude damps quickly even in the formation of the nonstationary torus of mass $\sim 0.1 M_{\odot}$. This is due to the fact that the compactness and degree of nonaxisymmetry of the torus decrease in a very short time scale $(\sim 2-3 \mathrm{~ms})$. Moreover, the gravitational wave amplitude of the QNM is very small. This implies that the amplitude of the Fourier spectrum steeply decreases in the high-frequency region for $f \gtrsim 1 / 0.8 \mathrm{~ms} \approx 1.2 \mathrm{kHz}$ (see Sec. IV G] cf. Fig. (7).

Gravitational waveforms are qualitatively similar for other models. However, quantitative differences due to the difference of the NS radius are clearly seen. Figure 6 (c) plots gravitational waves for model C (solid curves) together with those for model A (dashed curves). For $t_{\text {ret }} \lesssim 4 \mathrm{~ms}$, the inspiral waveforms for two models agree very well, because gravitational waves at such phase are primarily determined by the total mass and mass ratio. However, for $t_{\text {ret }} \gtrsim 4 \mathrm{~ms}$, the waveforms disagree because the amplitude for model $\mathrm{C}$ starts decreasing (compare the waveforms for $4.5 \mathrm{~ms} \lesssim t_{\text {ret }} \lesssim 6 \mathrm{~ms}$ ). This 


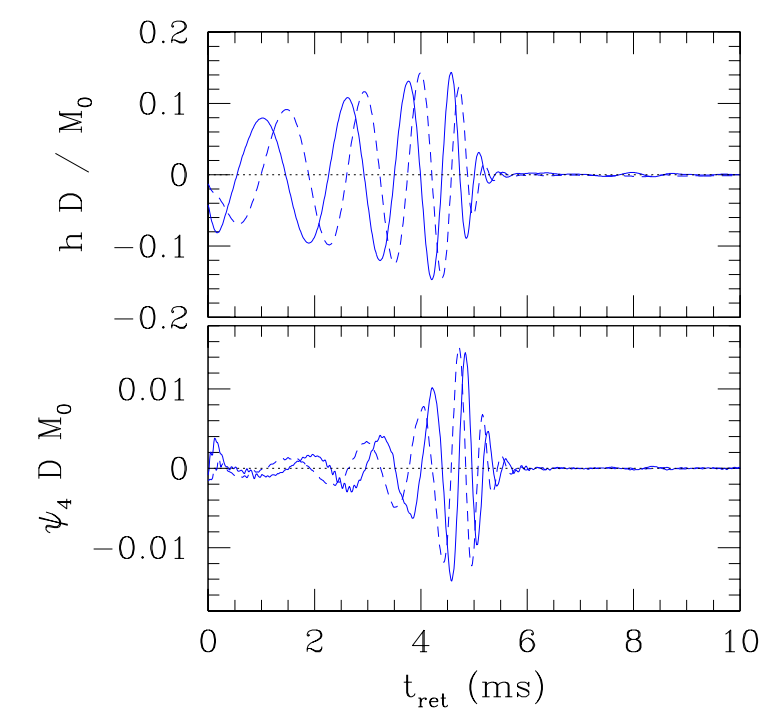

(a)

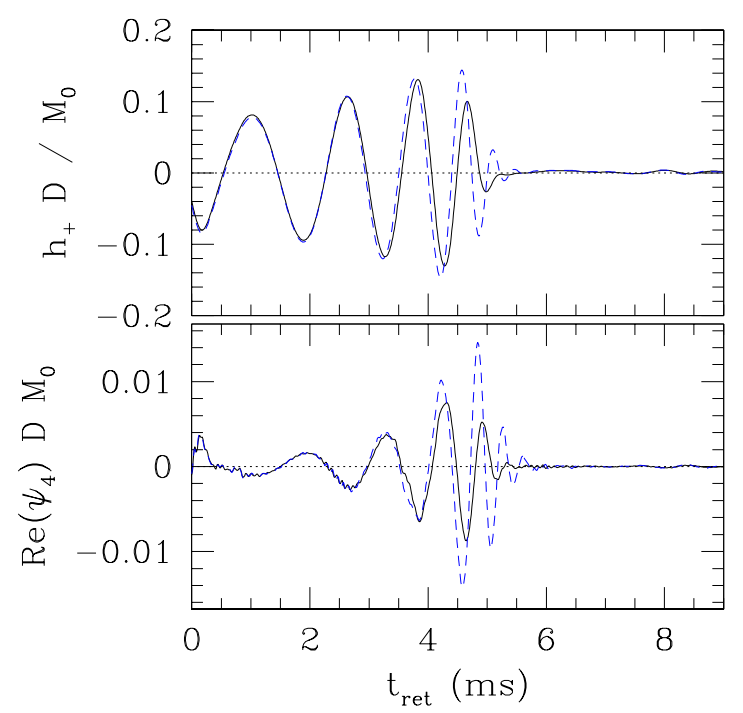

(c)

FIG. 6: (a) upper panel: + and $\times$ modes of gravitational waveforms observed along the $z$-axis for model A (solid and dashed curves, respectively). $t_{\text {ret }}$ denotes the retarded time [see Eq. (21)] and $M_{0}$ is the total mass defined in the caption of Table I. The amplitude at a distance of observer can be found from Eq. (22). (a) lower panel: The real and imaginary parts of $\psi_{4}$ (solid and dashed curves). (b) The real (upper panel) and imaginary (lower panel) parts of $\psi_{4}$ for model A together with the hypothetical curves of gravitational waves associated with the QNM (dotted curves) for 5 ms $\lesssim t_{\text {ret }} \lesssim 6.5$ ms. (c) $h_{+}$(upper panel) and $\operatorname{Re}\left(\psi_{4}\right)$ (lower panel) for models A (dashed curve) and C (solid curve). (d) The real (solid curve) and imaginary (dashed curve) parts of $\psi_{4}$ for model B (upper panel). The waveforms for $4.8 \mathrm{~ms} \lesssim t_{\text {ret }} \lesssim 6$ ms are magnified in the lower panel together with the hypothetical curves of gravitational waves associated with the QNM (dotted curves). For all the plots, only the $l=|m|=2$ modes are taken into account.

earlier decrease results from the onset of tidal disruption of the NS at a larger orbital separation due to the larger NS radius for model C (see Eq. 44). Soon after the onset of tidal disruption, the material spreads around the $\mathrm{BH}$ at a relatively large orbital separation. Because the compactness and degree of nonaxisymmetry decrease as the tidally disrupted material spreads, the amplitude for

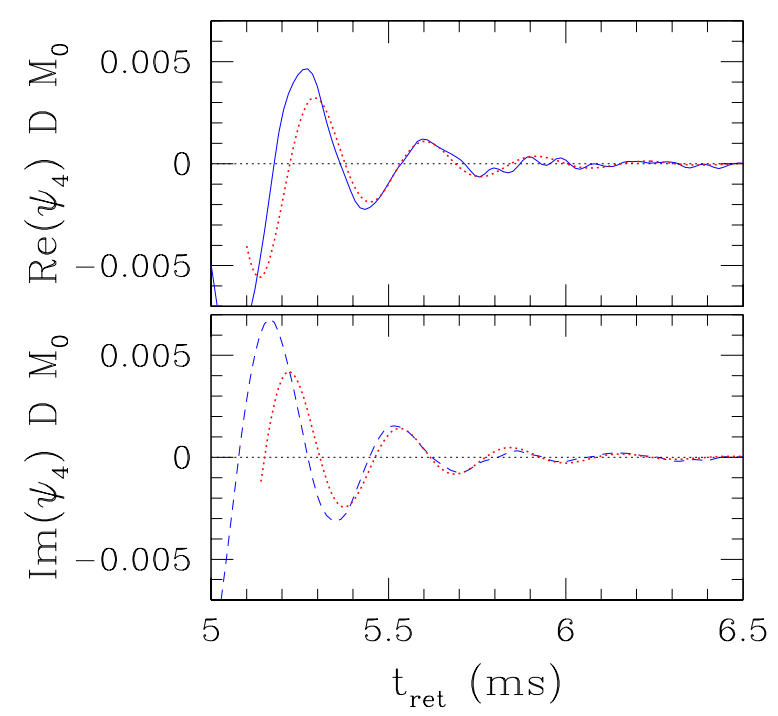

(b)
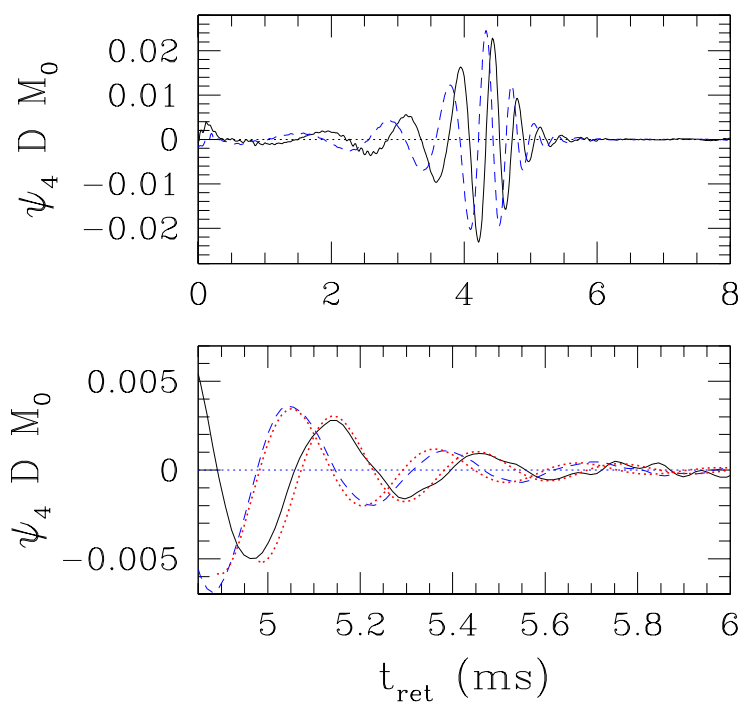

(d) 
By contrast, the QNM is clearly seen for the case that the NS radius is smaller. Figure 6(d) plots the $l=|m|=2$ mode of $\psi_{4}$ for model B. In this case, the tidal disruption sets in at an orbit closer to the ISCO than that for models $\mathrm{A}$ and $\mathrm{C}$. Consequently, a larger fraction of the material coherently falls into the $\mathrm{BH}$, and relatively coherently excites the QNM. In the lower panel of Fig. 6(d), we again fit the waveforms by Eq. (25) with $f_{\mathrm{qnm}}=3.23 \mathrm{kHz}$ and $t_{d}=0.289 \mathrm{~ms}$ assuming that the $\mathrm{BH}$ mass and spin are $5.12 M_{\odot}$ and 0.57 , respectively. Figure 6 (d) shows that the waveforms for $t_{\text {ret }} \gtrsim 5 \mathrm{~ms}$ agree well with the hypothetical fitting formula. A distinguishable feature is that the amplitude of the QNM is by a factor of $\sim 2$ larger than that for model A. However, this amplitude is still much smaller than the amplitude at the last inspiral orbit.

The kick velocity is also estimated from the total linear momentum carried by gravitational waves. We find that it is $\lesssim 20 \mathrm{~km} / \mathrm{s}$ for all the models (see Table III) [53]. This value is much smaller (by one order of magnitude) than that in the case of the $\mathrm{BH}-\mathrm{BH}$ merger [38]. The reason for this is that the gravitational wave amplitude damps quickly during the merger due to the tidal disruption, and the amplitude associated with the QNM is very small. Actually, a PN study [46] illustrates that the kick velocity is $\approx 20 \mathrm{~km} / \mathrm{s}$ for $q=1 / 3$ if the kick only by gravitational waves from the inspiral motion is taken into account. This value agrees fairly with our results. In the $\mathrm{BH}-\mathrm{BH}$ merger, gravitational waves associated with the QNM have a large amplitude and as a result, the linear momentum flux is significantly enhanced. If the NS escapes the tidal disruption during the merger (i.e., for the case that the $\mathrm{BH}$ mass is large enough or the NS radius is small enough), a large kick velocity of order $100 \mathrm{~km} / \mathrm{s}$ might be induced.

\section{G. Gravitational wave spectrum}

To determine the characteristic frequency of gravitational waves emitted during the tidal disruption, we compute the Fourier spectrum of gravitational waves of $l=|m|=2$ modes defined by

$$
h(f) \equiv \frac{D}{M_{0}} \sqrt{\frac{\left|h_{+}(f)\right|^{2}+\left|h_{\times}(f)\right|^{2}}{2}},
$$

where

$$
\begin{aligned}
& h_{+}(f)=\int e^{2 \pi i f t} h_{+}(t) d t, \\
& h_{\times}(f)=\int e^{2 \pi i f t} h_{\times}(t) d t .
\end{aligned}
$$

In the present simulations, we followed the inspiral phase only for about one and half orbits before the onset of tidal disruption. Thus, the Fourier spectrum for the low-frequency region is absent if we perform the Fourier transformation for the purely numerical data (see the dashed curve of Fig. 7(a)). To artificially compensate the Fourier spectrum for the low-frequency region, we combine the hypothetical waveforms computed from the third PN approximation for two structureless, inspiraling compact objects [6] . Specifically we determine the evolution of the orbital angular velocity $\Omega$ using the so-called Taylor T4 formula (e.g. 32] for a detailed review) by solving

$$
\begin{aligned}
& \frac{d x}{d t}=\frac{64 \eta x^{5}}{5 M_{0}}\left[1-\frac{743+924 \eta}{336} x+4 \pi x^{3 / 2}\right. \\
&+\left(\frac{34103}{18144}+\frac{13661 \eta}{2016}+\frac{59 \eta^{2}}{18}\right) x^{2} \\
&- \frac{4159+15876 \eta}{672} \pi x^{5 / 2} \\
&+\left\{\frac{16447322263}{139708800}-\frac{1712 \gamma_{E}}{105}+\frac{16 \pi^{2}}{3}\right. \\
&+\left(\frac{-56198689}{217728}+\frac{451}{48} \pi^{2}\right) \eta+\frac{541}{896} \eta^{2} \\
&\left.-\frac{5605}{2592} \eta^{3}-\frac{856}{105} \log (16 x)\right\} x^{3} \\
&+\left.\left.\left(\frac{-4415}{4032}+\frac{358675}{6048} \eta+\frac{91495}{1512} \eta^{2}\right) \pi x^{7 / 2}\right] 29\right)
\end{aligned}
$$

where $x=\left[M_{0} \Omega(t)\right]^{2 / 3}$ is a function of time [54], $\eta$ is the ratio of the reduced mass to the total mass $M_{0}$, and $\gamma_{E}=0.577 \cdots$ is the Euler constant. Then, gravitational waveforms are determined from

$$
\begin{aligned}
& h_{+}(t)=\frac{4 \eta M_{0} x}{D} A(x) \cos [\Phi(t)+\delta], \\
& h_{\times}(t)=\frac{4 \eta M_{0} x}{D} A(x) \sin [\Phi(t)+\delta],
\end{aligned}
$$

where $A(x)$ is a nondimensional function of $x$ for which $A(x) \rightarrow 1$ for $x \rightarrow 0, \delta$ is an arbitrary phase, and

$$
\Phi(t)=2 \int \Omega(t) d t .
$$

For $A(x)$, we choose the 2.5 PN formula (e.g., 32]). The PN waveforms are calculated for $M_{0} \Omega \geq 0.005$.

In this method, it is not clear at which frequency we should combine the PN waveforms with the numerical waveforms. In the present paper, we match the two waveforms at $M_{0} \Omega \approx 0.04$ (at $f \approx 517\left(M_{0} / 5 M_{\odot}\right)^{-1} \mathrm{~Hz}$ ). The Fourier spectrum for the late inspiral phase depends on this matching frequency, in particular, around the matching frequency $\sim 500-700 \mathrm{~Hz}$. However, the spectrum around the frequency for $f \gtrsim f_{\text {tidal }}$, for which the tidal disruption proceeds, depends very weakly on the matching frequency. Thus, the present method is acceptable for studying the Fourier spectrum during the tidal disruption.

Figure 7(a) plots $h(f) f$ for model A. We also plot the spectra for the analytical third PN waveforms (dotted curve) and for the purely numerical waveforms with no 

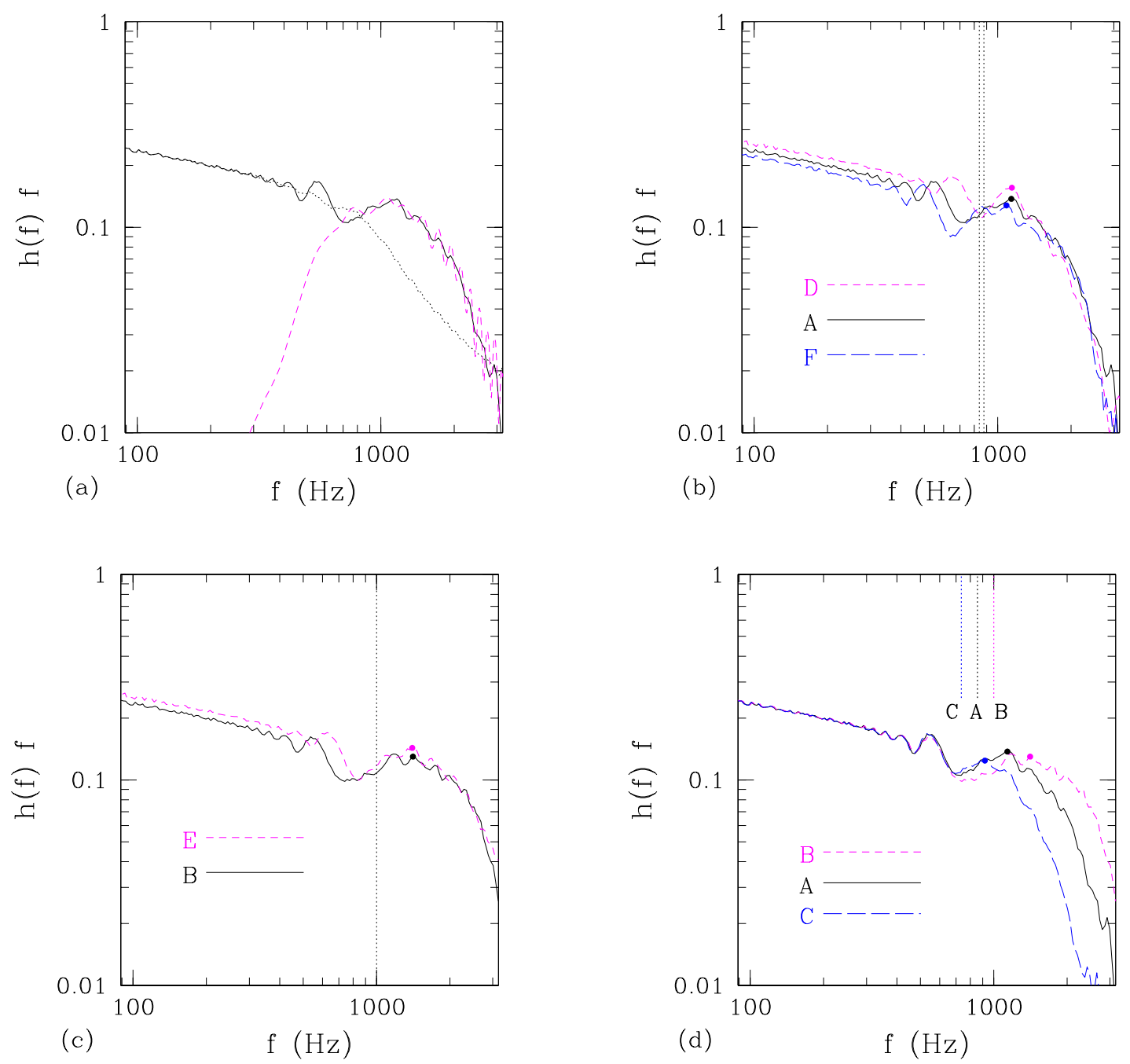

FIG. 7: (a) Spectrum of gravitational waves $h(f) f$ for model A (solid curve). The dashed and dotted curves are the spectra for the purely numerical (short-term) data and for the analytic third PN waveform. (b) the same as (a) but for models A, $\mathrm{D}$, and $\mathrm{F}$. The vertical dotted lines denote the expected frequencies at which the tidal disruption sets in for models $\mathrm{D}$ and $\mathrm{F}$; $f_{\text {tidal }} \approx 0.84 \mathrm{kHz}$ for model $\mathrm{F}$ and $0.88 \mathrm{kHz}$ for model $\mathrm{D}$. (c) the same as (b) but for models $\mathrm{D}$ and E. The vertical dotted line denotes the expected frequency at which the tidal disruption sets in, $f_{\text {tidal }} \approx 1.0 \mathrm{kHz}$. (d) the same as (b) but for models A-C. For (b)-(d), the solid circles denote the location of $f_{\text {cut }}$.

matching (dashed curve). For $\pi M_{0} f \ll 1$, the spectra of the matched and PN waveforms behave [7]

$$
h(f) f=\sqrt{\frac{5}{24 \pi}} \eta^{1 / 2}\left(\pi M_{0} f\right)^{-1 / 6} .
$$

We note that the effective amplitude defined by the average over the source direction and the direction of the binary orbital plane is

$$
\begin{aligned}
h_{\mathrm{eff}} \equiv \frac{2}{5} \frac{h(f) f M_{0}}{D} & \\
= & 9.6 \times 10^{-23}\left(\frac{h(f) f}{0.1}\right)\left(\frac{M_{0}}{5 M_{\odot}}\right) \\
& \times\left(\frac{D}{100 \mathrm{Mpc}}\right)^{-1} .
\end{aligned}
$$

Thus, the effective amplitude is $\approx 10^{-22}$ at $f \approx 1 \mathrm{kHz}$ for $D=100 \mathrm{Mpc}$ and $M_{0}=5 M_{\odot}$.

It is found that the spectrum for the purely numerical data agrees with the spectrum of the matched data for $f \gtrsim 800 \mathrm{~Hz}$ besides the spurious modulation for the spectrum of the purely numerical data which results from the incomplete data sets for the inspiral phase. Here, $f=800 \mathrm{~Hz}$ is slightly smaller than $f_{\text {tidal }}$ predicted from Eq. (5) (see also Table IV). This illustrates that the matching does not affect the global shape of the spectrum for $f \gtrsim f_{\text {tidal }}$.

We still see the modulation and dip of the spectrum for the matched data in the frequency band between $\sim 500$ and $800 \mathrm{~Hz}$. The possible physical reason for the dip at $f \lesssim 1 \mathrm{kHz}$ is that the radial-approach velocity of the $\mathrm{BH}$ 
and NS steeply increases near the ISCO $(f \sim 1.2 \mathrm{kHz})$, and hence, the integration time for gravitational waves decreases. As a result, the effective amplitude at such high-frequency band slightly decreases. This feature is well-known for the NS-NS merger [47]. However, there are also the possible unphysical reasons as follows: (i) the orbit of the BH-NS has a nonzero eccentricity because the initial data is not exactly the circular orbit [48], and (ii) the matching of the numerical and PN data induces a systematic error. Thus, the spectrum for this band may not be very accurate. We do not pay attention to this low-frequency band in the following.

The noteworthy feature of the spectrum is that the spectrum amplitude does not damp even for $f_{\text {tidal }}<f \lesssim$ $1.3 f_{\text {tidal }} \equiv f_{\text {cut }}$ (see the solid circles in Fig. [7(b)-(d) for the approximate location of $f_{\text {cut }}$ ). The reason for this is that the NS orbiting near the ISCO has a radialapproach velocity of order $\sim 10 \%$ of the orbital velocity due to the radiation reaction of gravitational waves, and hence, the NS is not immediately tidally disrupted at the orbit of $f=f_{\text {tidal }}$, although the tidal disruption of the NS likely sets in at $f=f_{\text {tidal }}$. As a result, the inspiral orbit is maintained for a while even inside the predicted tidal disruption orbit and gravitational waves of a large amplitude are emitted for $f>f_{\text {tidal }}$. Because the tidal disruption completes at $f \approx f_{\text {cut }}>f_{\text {tidal }}$, it is not straightforward to determine $f_{\text {tidal }}$ from the spectrum of gravitational waves.

We note that our finding of $f_{\text {cut }}>f_{\text {tidal }}$ is the unique feature for the BH-NS binary. If the companion of the $\mathrm{BH}$ is not as compact as the NS (e.g., for the BH-white dwarfs binary), $f_{\text {cut }}$ would be approximately equal to $f_{\text {tidal }}$ because the radial-approach velocity due to the gravitational radiation reaction is negligible and hence the tidal disruption would complete at the predicted tidal disruption orbit.

Because the amplitude of gravitational waves quickly decreases after the tidal disruption completes, the spectrum also sharply falls for the high-frequency band; i.e., above a cut-off frequency $f_{\text {cut }}$, the spectrum amplitude steeply decreases. Figure 7(a) shows that for model A, the value of $f_{\text {cut }}$ is approximately $1.16 \mathrm{kHz}$ (i.e., $M_{0} \Omega \approx 0.094$ ). This confirms the finding in Sec. IVF about the quick decrease of the gravitational wave amplitude occurs near the ISCO in this case.

To see the dependence of the spectrum shape on the mass ratio and NS radius, we plot Fig. 7(b)-(d). Figure 7(b) and (c) compare the spectra for models A, D, and $\mathrm{F}\left(R_{\mathrm{NS}}=13.2 \mathrm{~km}\right)$ and for models $\mathrm{B}$ and $\mathrm{E}\left(R_{\mathrm{NS}}=\right.$ $12.0 \mathrm{~km}$ ), for which the NS radii are identical each other, whereas the mass ratio $q$ is different. It is found that the spectrum shape depends weakly on the mass ratio. The reason for this is that the gravitational wave frequency at which the tidal disruption occurs is determined primarily by the radius and mass of the NS. For models A, D, and $\mathrm{F}, f_{\text {cut }} \sim 1.1-1.2 \mathrm{kHz}$, and for models $\mathrm{B}$ and $\mathrm{E}, f_{\text {cut }} \sim$ $1.4 \mathrm{kHz}$ (see Table IV). Obviously, for the smaller radii, the value of $f_{\text {cut }}$ is larger because the tidal disruption
TABLE IV: The expected frequency of gravitational waves at the tidal disruption $f_{\text {tidal }}$, the peak frequency of gravitational wave spectrum near the sharp cut-off in the high-frequency region $f_{\text {cut }}$, and the ratio $f_{\text {cut }} / f_{\text {tidal }}$.

\begin{tabular}{cccc}
\hline Model & $f_{\text {tidal }}(\mathrm{kHz})$ & $f_{\text {cut }}(\mathrm{kHz})$ & $f_{\text {cut }} / f_{\text {tidal }}$ \\
\hline $\mathrm{A}$ & 0.856 & 1.16 & 1.36 \\
\hline $\mathrm{B}$ & 0.997 & 1.41 & 1.41 \\
\hline $\mathrm{C}$ & 0.736 & 0.92 & 1.25 \\
\hline $\mathrm{D}$ & 0.877 & 1.14 & 1.30 \\
\hline $\mathrm{E}$ & 1.021 & 1.40 & 1.37 \\
\hline $\mathrm{F}$ & 0.840 & 1.09 & 1.30 \\
\hline
\end{tabular}

completes for the smaller orbital separation.

We also calculate the ratio of $f_{\text {cut }}$ to $f_{\text {tidal }}$ (see Table IV). For models A, D, and F, the ratio is $\sim 1.3$, whereas for models $\mathrm{B}$ and $\mathrm{E}$, it is slightly larger, $\sim 1.4$. The reason is that the compact NS has the larger radial-approach velocity at $f=f_{\text {tidal }}$, and hence, the tidal disruption completes at a smaller orbital separation.

Figure 7(d) compares the spectra for models A-C, for which the mass ratio $q$ is approximately identical, whereas the NS radii are different. We find that the value of $f_{\text {cut }}$ depends strongly of the NS radius and is smaller for larger NS radii, indicating that the tidal disruption sets in at a larger orbital separation. The ratio $f_{\text {cut }} / f_{\text {tidal }}$ is also smaller for larger NS radii (see Table IV). The reason for this is that for the larger NS, the tidal disruption sets in at a larger orbital separation, and hence, the radial-approach velocity is smaller. However, even for model $\mathrm{C}$, the ratio is $\approx 1.25$. This implies that gravitational waves of a high amplitude are emitted well inside the orbit of the onset of tidal disruption, even for a less compact NS of nearly upper-limit radius $\sim 15 \mathrm{~km}$.

Finally, we note the following: Figure 7 and Equation (34) show that the effective amplitude at $f=f_{\text {cut }} \sim 1$ $\mathrm{kHz}$ is $\approx 10^{-22}$ for the typical distance and total mass as $D=100 \mathrm{Mpc}$ and $M_{0}=5 M_{\odot}$. Even for the optimistic direction of the source and its binary orbital plane, the effective amplitude is at most $\approx 2.5 \times 10^{-22}$. The designed sensitivity of the advanced LIGO is $\sim 3 \times 10^{-22}$ [49]. This implies that it will not be possible to detect gravitational waves during the tidal disruption by the detectors of standard design. To detect gravitational waves at such high frequency, the detectors of special instrument (e.g., resonant-side band extraction [50]) which is sensitive to high-frequency gravitational waves is necessary.

\section{SUMMARY}

We have presented the numerical results of fully general relativistic simulation for the merger of BH-NS binaries, focusing on the case that the NS is tidally disrupted by a nonspinning low-mass $\mathrm{BH}$ of $M_{\mathrm{BH}} \approx 3.3-4.6 M_{\odot}$. The $\Gamma$-law EOS with $\Gamma=2$ and irrotational velocity field 
are employed for modeling the NSs. To see the dependence of the tidal disruption event on the NS radius, we choose it in a wide range as $R_{\mathrm{NS}}=12.0-1.47 \mathrm{~km}$ whereas the NS mass is fixed to be $\approx 1.3 M_{\odot}$. The resulting mass ratio $q \equiv M_{\mathrm{NS}} / M_{\mathrm{BH}}$ is in the range between $\approx 0.28$ and 0.4 .

As predicted by the study for the quasicircular states [3, 4], for all the chosen models, the NS is tidally disrupted at the orbits close to the ISCO. The BH of the spin of $0.5-0.6$ is formed and a large fraction of the material is quickly swallowed into the $\mathrm{BH}$, whereas $2-12 \%$ of the material forms a hot and compact torus around the $\mathrm{BH}$. The resultant mass and density of the torus depend strongly on the mass ratio $q$ and NS radius $R_{\mathrm{NS}}$, in particular on $R_{\mathrm{NS}}$. For $R_{\mathrm{NS}}=12.0 \mathrm{~km}$, the torus mass is at most $0.05 M_{\odot}$ even for the large value of the mass ratio $q \approx 0.39$. For $R_{\mathrm{NS}}=14.7 \mathrm{~km}$, by contrast, the torus mass is $\approx 0.16 M_{\odot}$ for $q \approx 0.33$. Extrapolating the results in this paper, the torus mass would be smaller than $0.01 M_{\odot}$ for $R_{\mathrm{NS}}=11 \mathrm{~km}$ even for $q \sim 0.4$. The stiff nuclear EOSs predict the radius of $\approx 11-12$ $\mathrm{km}$ for $M_{\mathrm{NS}}=1.3-1.4 M_{\odot}[29]$. This suggests that the torus mass is likely to be $\ll 0.1 M_{\odot}$ even for the $\mathrm{BH}$ mass $3-4 M_{\odot}$. This point should be more rigorously clarified for the future simulation employing the realistic nuclear EOSs.

For the optimistic cases in which $R_{\mathrm{NS}} \gtrsim 13 \mathrm{~km}$ or of $q \gtrsim 0.4$, the torus mass can be $\gtrsim 0.05 M_{\odot}$. As we found from the value of the $\varepsilon_{\mathrm{th}}$, the resultant torus likely has high temperature as $10^{10}-10^{11} \mathrm{~K}$. This suggests that such outcomes are promising candidates for driving SGRBs. According to the latest simulations for the hot and compact torus around the BH by Setiawan et al. [41], the total energy deposited by neutrino-antineutrino annihilation is $\sim 10^{49}\left(M_{\text {torus }} / 0.01 M_{\odot}\right)$ ergs for $0.01 M_{\odot} \lesssim$ $M_{\text {torus }} \lesssim 0.1 M_{\odot}$ and $a=0.6$. Here, $M_{\text {torus }}$ is the initial torus mass for the simulation. Assuming that the conversion rate to the gamma-ray energy is $10 \%$ [51], the total energy of SGRBs is $\sim 10^{48}\left(M_{\text {torus }} / 0.01 M_{\odot}\right)$ ergs according to their numerical results. This indicates that if the NS radius is $\gtrsim 13 \mathrm{~km}$ with $M_{\mathrm{BH}}=3.3-4 M_{\odot}$, the SGRB of the total energy $\sim 10^{49}$ ergs may be driven. By contrast, for $R_{\mathrm{NS}} \lesssim 12 \mathrm{~km}$ with $M_{\mathrm{BH}} \gtrsim 3.3 M_{\odot}$, the total energy is likely to be at most several $\times 10^{48}$ ergs; i.e., only the weak SGRBs can be explained. We note that the final spin parameter depends on the initial spin of the $\mathrm{BH}$. In the merger of the rapidly rotating $\mathrm{BH}$ and NS, the resuling spin of the BH will be close to unity. For such BHs, the total deposited energy will be enhanced by the spin effects [43].

Gravitational waves are also computed. It is found that the amplitude of gravitational waves damps during the tidal disruption, and when the tidal disruption completes, the amplitude becomes much smaller than that at the last inspiral orbit. Although we find that the waveforms in the final phase of the tidal disruption are characterized by the QNM ringing, its amplitude is much smaller than that of gravitational waves at the last in- spiral phase, in particular, for the large value of $R_{\mathrm{NS}}$. The reason for this is that the NS is tidally disrupted before plunging into the $\mathrm{BH}$, and hence, a significant excitation of the QNM by the coherently infalling material is not achieved in contrast to the BH-BH merger (e.g., [18, 19, 30, 31]). For the case that the NS is compact with $R_{\mathrm{NS}}=12 \mathrm{~km}$, the tidal disruption occurs at an orbit very close to the ISCO and a large amount of the material falls fairly coherently into the $\mathrm{BH}$, resulting in a relatively high amplitude of the QNM. However, the amplitude is still much smaller than that at the last inspiral orbit.

The Fourier spectrum of gravitational waves is also analyzed. Because the amplitude of gravitational waves quickly damps during the tidal disruption, the spectrum amplitude steeply decreases above a cut-off frequency $f_{\text {cut }}$. The noteworthy feature is that the cut-off frequency does not agree with the predicted frequency at which the tidal disruption sets in, $f_{\text {tidal }}$. The reason for this is that the NS in the compact binaries has a radial-approach velocity of order $\sim 10 \%$ of the orbital velocity and is not immediately tidally disrupted at $f=f_{\text {tidal }}$. We have found that $f_{\text {cut }} / f_{\text {tidal }}$ is in the range between 1.25 and 1.4 in our results depending primarily on the NS radius $R_{\mathrm{NS}}$; this value is larger for the smaller values of $R_{\mathrm{NS}}$. These results imply that it is not straightforward to determine $f_{\text {tidal }}$ from gravitational wave observation. If the dependence of $f_{\text {cut }} / f_{\text {tidal }}$ on the NS radius and mass ratio is clarified in detail by the numerical simulation, $f_{\text {tidal }}$ may be inferred from $f_{\text {cut }}$. If such a relation is found, $f_{\text {cut }}$ will be useful for determining the properties of NSs such as the radius and density profile. For this purpose, a detailed simulation taking into account the nuclear EOSs is necessary. We plan to perform such simulation in the next step.

\section{Acknowledgments}

We deeply thank members in the Meudon relativity group, in particular, Eric Gourgoulhon, for developing the free library LORENE, which is used for computation of the initial conditions. Numerical computations were performed on the FACOM-VPP5000 at CfCA at National Astronomical Observatory of Japan and on the NEC-SX8 at Yukawa Institute of Theoretical Physics of Kyoto University. This work was supported by a Monbukagakusho Grant (No. 19540263).

\section{APPENDIX A: COMPARISON WITH THE LOW-RESOLUTION RESULTS}

In this appendix, we present the results for models A1, A2 and C2 for which the physical parameters of the initial conditions are the same as those for models A and $\mathrm{C}$ but the simulations were performed with the poorer grid resolutions (see Table II). 


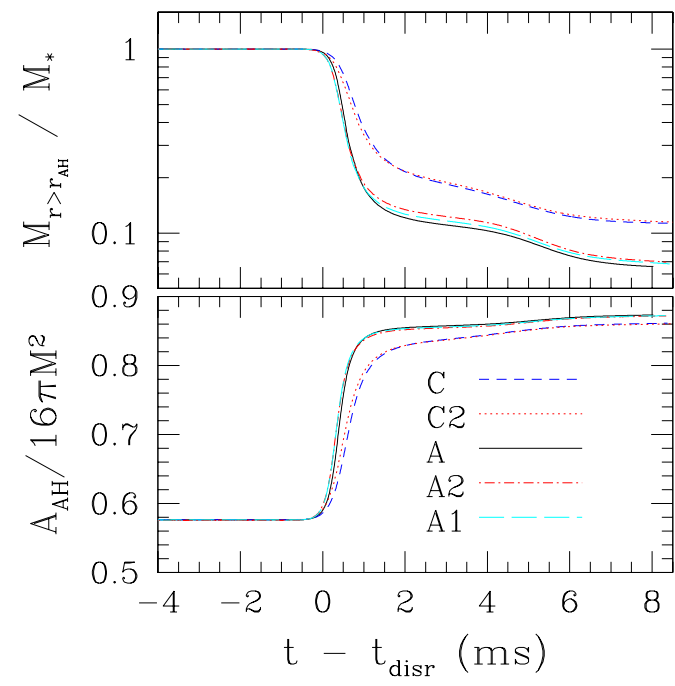

FIG. 8: The same as Fig. 5(c) but for models A, C, A1, A2, and $\mathrm{C} 2$. We choose the values of $t_{\mathrm{disr}}=4.30 \mathrm{~ms}, 4.17 \mathrm{~ms}$, and $4.50 \mathrm{~ms}$ for $\mathrm{A} 2, \mathrm{~A} 1$, and $\mathrm{C} 2$, respectively. For models A and $\mathrm{C}$, see the caption of Fig. 5 .

Figure 8 plots the evolution of the rest mass of the material located outside the apparent horizon $M_{r>r_{\mathrm{AH}}}$ and the area of the apparent horizon in units of $16 \pi M^{2}$ for models A1, A2, and C2. For comparison, the results for models $\mathrm{A}$ and $\mathrm{C}$ are shown together.

We find that the results for models A1 and A2 agree qualitatively well with those for model A. Because of the difference of the grid structure for covering the $\mathrm{BH}$ and NS and/or because of the larger numerical dissipation for the low-resolution runs, the time of the onset of tidal disruption $t_{\text {disr }}$ slightly disagrees among three models, but the difference is only $\approx 0.2-0.3 \mathrm{~ms}(\sim 10 \%$ of the orbital period at the last inspiral orbit). The value of $M_{r>r_{\mathrm{AH}}}$ $\left(A_{\mathrm{AH}} / 16 \pi M^{2}\right)$ is systematically overestimated (underestimated) for the lower-resolution runs. However, the magnitude of the disagreement is not large. For model A2 (A1), the relative errors of $M_{r>r_{\mathrm{AH}}}$ and $A_{\mathrm{AH}}$ to the values for model $\mathrm{A}$ is $\sim 7 \%(\sim 4 \%)$ and $\sim 0.3 \%(\sim 0.3 \%)$, respectively. Furthermore, the values systematically converge, although the order of the convergence is not exactly specified because the difference among three results is small. (Note that the order of the convergence in the presence (absence) of shocks should be the first (third) order for the hydro part whereas that for the gravitational field is fourth order, and thus, the order of the convergence is not simply determined). For determining the order of the convergence, it is necessary to perform simulations with a better accuracy. However, it is not feasible to do it in our present computational resources. Assuming that the second-order convergence holds for $M_{r>r_{\mathrm{AH}}}$, the extrapolation gives the exact value of $M_{r>r_{\mathrm{AH}}}$, and we find that the errors for $M_{r>r_{\mathrm{AH}}}$ at $t-t_{\mathrm{disr}}=7 \mathrm{~ms}$ is $\sim 0.019 M_{\odot}(\sim 22 \%), 0.024 M_{\odot}(\sim 30 \%)$, and $0.030 M_{\odot}$ $(\sim 35 \%)$ for models $\mathrm{A}, \mathrm{A} 1$, and $\mathrm{A} 2$, respectively. For $A_{\mathrm{AH}} / 16 \pi M^{2}$, the relative errors at $t-t_{\mathrm{disr}}=7 \mathrm{~ms}$ are $\sim 0.3 \%, 0.5 \%$, and $0.5 \%$ for models $\mathrm{A}, \mathrm{A} 1$, and $\mathrm{A} 2$.

We find that the results for model $\mathrm{C} 2$ agree well with those for model C. In this case, the time of the onset of tidal disruption $t_{\mathrm{disr}}$ agrees within $\approx 0.04 \mathrm{~ms}(\sim 2 \%$ of the orbital period at the last inspiral orbit). Furthermore, the value of $M_{r>r_{\mathrm{AH}}}$ and the area of the apparent horizon agree within $\sim 3 \%$ and $\sim 0.1 \%$ errors, respectively. Assuming the second-order convergence, the exact values of $M_{r>r_{\mathrm{AH}}}$ and $A_{\mathrm{AH}} / 16 \pi M^{2}$ at $t-t_{\mathrm{disr}}=8$ $\mathrm{ms}$ are determined, and by comparison with these values, we find that the errors of these values for model $\mathrm{C}$ $(\mathrm{C} 2)$ are $4 \%(6 \%)$ and $0.2 \%(0.4 \%)$, respectively. For model $\mathrm{C} 2$, the grid spacing for the inner computational domain is $\Delta x=M_{\mathrm{p}} / 12$, with which the major diameter of the NS is covered by the 50 grid points (see Table II). Thus, with such setting, the orbits of the BH and NS are computed with an accuracy enough at least for the qualitative study.

Figure 9 plots $h_{+}$and $h_{\times}$(a) for models A and A2 and (b) for models $\mathrm{C}$ and $\mathrm{C} 2$. This shows that the waveforms for the high and low-resolution runs agree qualitatively well. For the inspiral phase, the difference of the waveforms for the two different resolutions is very small. In particular, for models $\mathrm{C}$ and $\mathrm{C} 2$, the amplitude at a given time agrees each other within $\sim 3 \%$ error for $t_{\text {ret }} \lesssim 4.5$ ms. During the tidal disruption phase, the amplitude is systematically smaller for the low-resolution runs. The possible reason for this is that the compactness of the NS is more quickly lost with the lower resolution, resulting in the less coherent excitation of gravitational waves. However, the difference of the amplitude is still at most $\sim 10 \%$ for model C2. For model A2, the error is larger in particular for $4.5 \mathrm{~ms} \lesssim t_{\text {ret }} \lesssim 5 \mathrm{~ms}$, although the waveforms agree qualitatively with those for model A. The primary source of the error is the phase difference caused by the fact that for the lower resolution, the tidal disruption sets in earlier. During the tidal disruption, the wave amplitude for model A2 is by a factor of $\sim 2$ smaller than that for model A. This indicates that the simulation of the poor resolution significantly underestimates the amplitude during the tidal disruption phase. Because of the underestimation of the amplitude, the total energy and angular momentum emitted by gravitational waves are underestimated by $\sim 5 \%$ for model $\mathrm{C} 2$ and by $\sim 10 \%$ for model A2 in comparison with models $\mathrm{C}$ and $\mathrm{A}$.
[1] I. H. Stairs, Science 304, 547 (2004).

[2] R. Voss and T. M. Taulis, Mon. Not. R. Astro. Soc.
342, 1169 (2003); K. Belczynski, T. Bulik, and B. Rudak, Astrophys. J. 571, 394 (2002); V. Kalogera et 


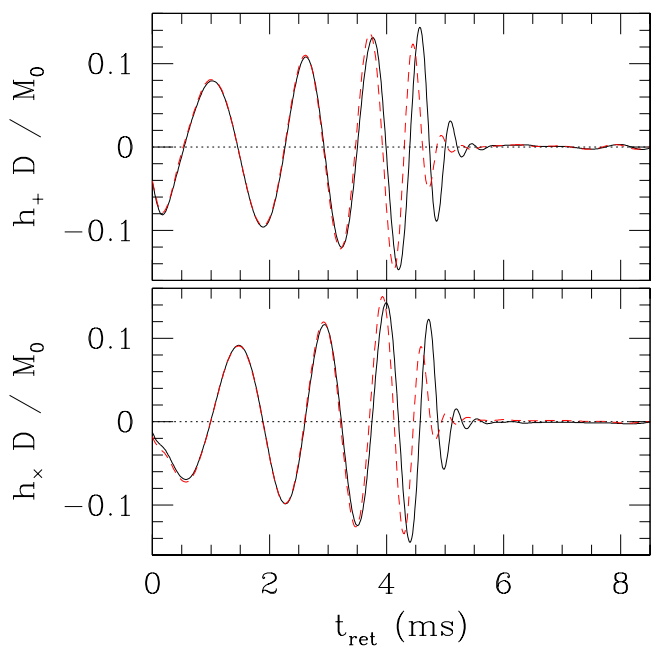

(a)

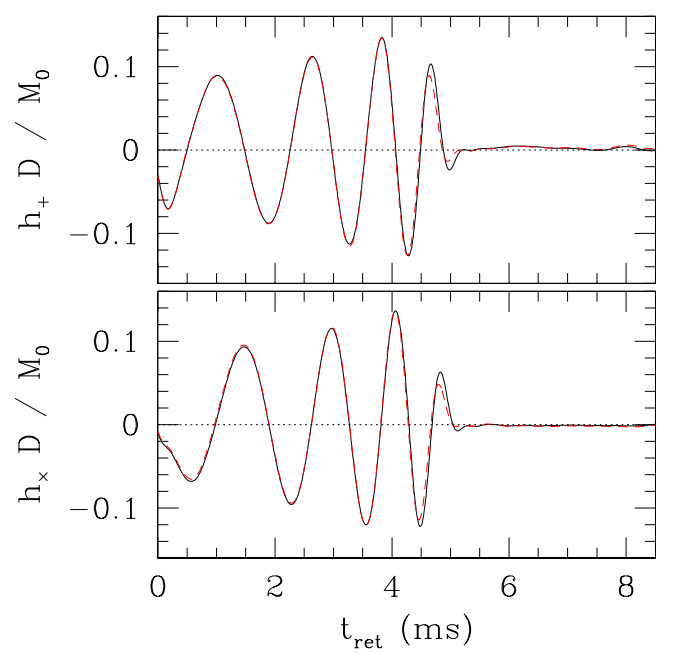

(b)

FIG. 9: The same as Fig. 6(c) but (a) for models A (solid curves) and A2 (dashed curves) and (b) for models C (solid curves) and $\mathrm{C} 2$ (dashed curves), respectively.

al. Astrophys. J. 601, L179 (2004); V. Kalogera et al., astro-ph/0612144

[3] K. Taniguchi, T. W. Baumgarte, J. A. Faber, and S. L. Shapiro, Phys. Rev. D 75, 084005 (2007).

[4] K. Taniguchi, T. W. Baumgarte, J. A. Faber, and S. L. Shapiro, submitted to Phys. Rev. D.

[5] M. Ishii, M. Shibata, and Y. Mino, Phys. Rev. D 71, 044017 (2005).

[6] L. Blanchet, Living Rev. Relativ. Vol. 9, No. 4 (2006).

[7] E.g., C. Cutler and E. E. Flanagan, Phys. Rev. D 49, 2658 (1994).

[8] L. Lindblom, Astrophys. J. 398, 569 (1992).

[9] M. Vallisneri, Phys. Rev. Lett. 84, 3519 (2000).

[10] C. L. Fryer, S. E. Woosley, M. Herant, and M. B. Davies, Astrophys. J. 520, 650 (1999).

[11] E. Barger, in AIP Conf. Proc. 836, 33 (2006).

[12] W. H. Lee and W. Kluzniak, Astrophys. J. 526, 178 (1999); H. T. Janka, T. Eberl, M. Ruffert, and C. L. Fryer, Astrophys. J. 527, L39 (1999).

[13] J. A. Faber, T. W. Baumgarte, S. L. Shapiro, and K. Taniguchi, Astrophys. J. 641, L93 (2006).

[14] J. A. Faber, T. W. Baumgarte, S. L. Shapiro, K. Taniguchi, and F. A. Rasio, Phys. Rev. D 73, 024012 (2006).

[15] M. Shibata and K. Uryū, Phys. Rev. D 74, 121503 (R) (2006); Class. Quantum Grav. 24, S125 (2006).

[16] M. Shibata, K. Taniguchi, and K. Uryū, Phys. Rev. D 68, 084020 (2003).

[17] M. Shibata, K. Taniguchi, and K. Uryū, Phys. Rev. D 71, 084021 (2005); M. Shibata and K. Taniguchi, ibid 73, 064027 (2006).

[18] M. Campanelli, C. O. Lousto, P. Marronetti, and Y. Zlochower, Phys. Rev. Lett. 96, 111101 (2006); J. G. Baker, J. Centrella, D.-I. Choi, M. Koppitz, and J. van Meter, Phys. Rev. Lett. 96, 111102 (2006).

[19] B. Brügmann, J. A. Conzalez, M. Hannam, S. Husa, and U. Sperhake, gr-qc/0610128

[20] C. S. Kochanek, Astrophys. J. 398, 234 (1992); L. Bildsten and C. Cutler, ibid 400, 175 (1992).
[21] M. Shibata, Phys. Rev. D 58, 024012 (1998); S. A. Teukolsky, Astrophys. J. 504, 442 (1998).

[22] E. Gourgoulhon, P. Grandclément, K. Taniguchi, J.-A. Marck, and S. Bonazzola, Phys. Rev. D 63, 064029 (2001).

[23] K. Taniguchi and E. Gourgoulhon, Phys. Rev. D 66, 104019 (2002): ibid 68, 124025 (2003).

[24] LORENE website: http://www.lorene.obspm.fr/

[25] P. Grandclément, Phys. Rev. D 74, 124002 (2006) [Erratum ibid. 75, 129903 (2007)].

[26] K. Taniguchi, T. W. Baumgarte, J. A. Faber, and S. L. Shapiro, Phys. Rev. D 72, 044008 (2006).

[27] Z. B. Etienne, J. A. Faber, Y.T. Liu, S. L. Shapiro, K. Taniguchi, and T. W. Baumgarte, in preparation.

[28] J. M. Lattimer and M. Prakash, Science 304, 536 (2004).

[29] E.g., A. Akmal, V. R. Pandharipande, and D. G. Ravenhall, Phys. Rev. C 58, 1804 (1998); F. Douchin and P. Haensel, Astron. Astrophys. 380, 151 (2001).

[30] A. Buonanno, G. B. Cook, and F. Pretorius, Phys. Rev. D 75, 124018 (2007).

[31] J. G. Baker, et al., Phys. Rev. D 75, 124024 (2007); M. Hannam, H. Husa, U. Sperhake, B. Brügmann, and J. A. Gonzalez, gr-qc/0706.1305.

[32] M. Boyle et al., gr-qc/0710.0158.

[33] M. Shibata and J. A. Font, Phys. Rev. D 72, 047501 (2005).

[34] M. Shibata, Phys. Rev. D 67, 024033 (2003).

[35] M. Shibata and T. Nakamura, Phys. Rev. D 52, 5428 (1995).

[36] V. Moncrief, Ann. of Phys. 88, 323 (1974).

[37] M. Shibata and Y.I. Sekiguchi, Phys. Rev. D 71, 024014 (2005).

[38] J. G. Baker, et al. Astrophys. J. Lett. 653, 93 (2006); J. A. Gonzalez et al., Phys. Rev. Lett. 98, 091101 (2007); L. Rezzolla, et al., gr-qc/0708.3999, and references cited therein.

[39] M. Shibata, Phys. Rev. D 55, 2002 (1997): M. Shibata and K. Uryū, Phys. Rev. D 62, 087501 (2000).

[40] R. Popham, S. E. , and C. Fryer, Astrophys. J. 518 
(1999), 356; R. Narayan, T. Piran, and P. Kumar, Astrophys. J. 557 (2001), 949; T. DiMatteo, R. Perna, and R. Narayan, Astrophys. J. 579 (2002), 706; K. Kohri and S. Mineshige, Astrophys. J. 577 (2002), 311; A. Janiuk, R. Perna, T. DiMatteo, and B. Czerny, Mon. Not. R. Astron. Soc. 355 (2004), 950; W.-X. Chen and A. M. Beloborodov, Astrophys. J. 657 (2007), 383.

[41] S. Setiawan, M. Ruffert, and H.-Th. Janka, Mon. Not. R. Astron. Soc. 352 (2004), 753; Astron. Astrophys. 458 (2006), 553.

[42] W. H. Lee, E. Ramirez-Ruiz, and D. Page, Astrophys. J. 632 (2005), 421.

[43] M. Shibata, Y.I. Sekiguchi, and R. Takahashi, Prog. Theor. Phys. 118, 257 (2007).

[44] M. Shibata, Phys. Rev. D 76, 064035 (2007).

[45] E. W. Leaver, Proc. R. Soc. Lond. A 402, 285 (1985); F. Echeverria, Phys. Rev. D 40, 3194 (1989).

[46] L. Blanchet, M. S. S. Qusailar, and C. M. Will, Astrophys. J. 635, 508 (2005).

[47] E.g., X. Zhuge, J. M. Centrella, and S. L. W. McMillan, Phys. Rev. D 50, 6247 (1994).

[48] E. Berti, S. Iyer, and C. M. Will, gr-qc/0709.2589.

[49] K. S. Thorne, in Compact Stars in binaries: the pro- ceeding of IAU symposium No. 165 (Kluwer Academic Publishehes, 1996), pp. 153.

[50] S. Kawamura, private communication.

[51] E.g., M. A. Aloy, H.-T. Janka, and E. Müller, Astron. Astrophys. 436, 273 (2005).

[52] In this formula, we ignore the binding energy between the $\mathrm{BH}$ and surrounding material. Thus, $M_{\mathrm{BH}, \mathrm{f}}$ likely overestimates the true $\mathrm{BH}$ mass slightly.

[53] The studies for the kick velocity in the numerical relativity [38] has clarified that the numerical results of its magnitude depend sensitively on the initial condition. For the initial condition of small orbital separation, the given quasicircular orbit includes nonrealistic radial velocity and/or nonzero ellipticity. Due to such unrealistic elements, the estimated linear momentum flux includes an systematic error. The results of [38] show that the systematic error could be as large as the magnitude of the kick velocity. Because the simulation is started from a fairly close orbit in this work, the systematic error may be as large as the magnitude for the kick velocity.

[54] In this subsection, $x$ is different from the one of the Cartesian coordinates. 


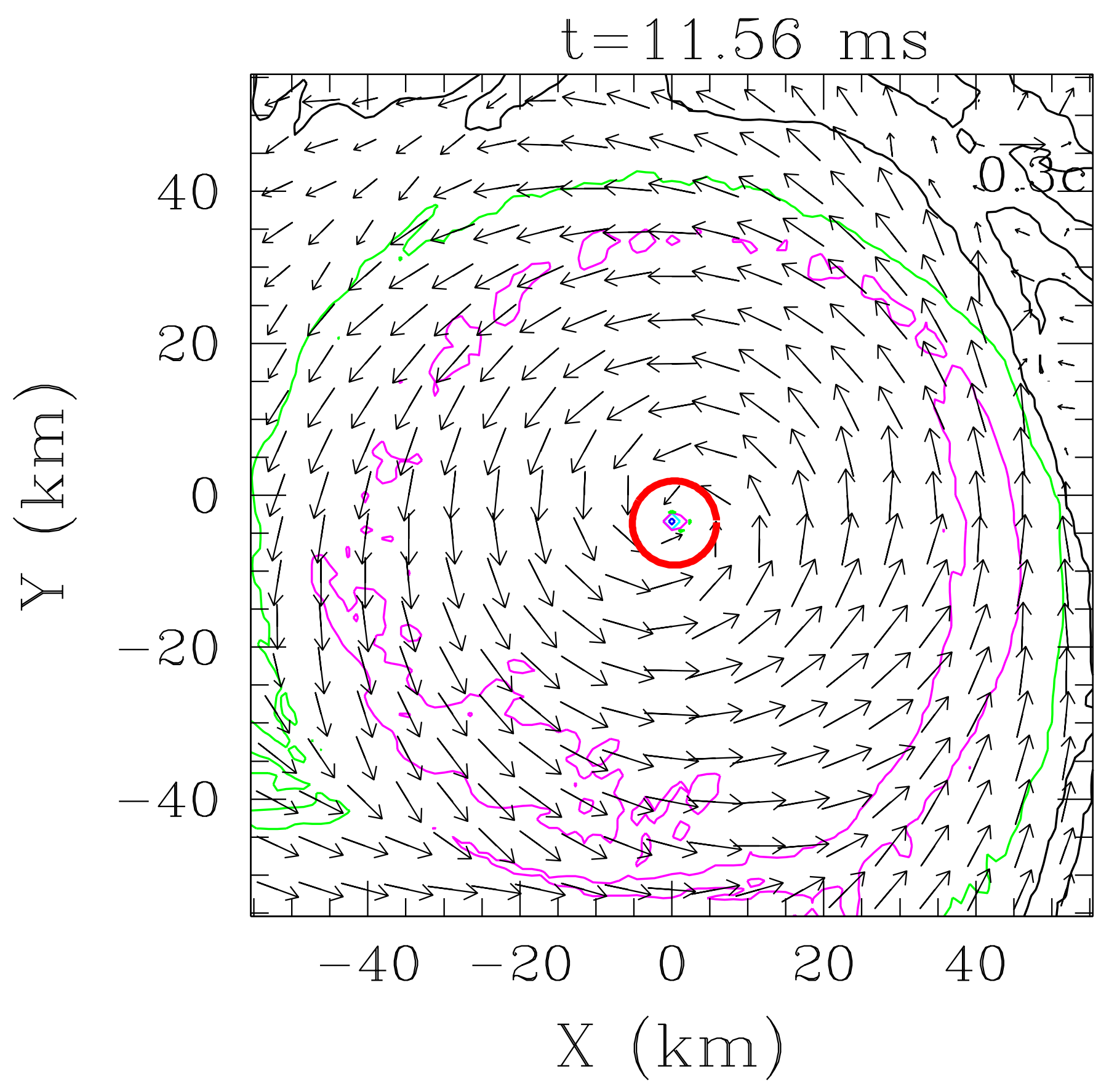

